\title{
ON THE INTERPRETATION OF WHITNEY NUMBERS THROUGH ARRANGEMENTS OF HYPERPLANES, ZONOTOPES, NON-RADON PARTITIONS, AND ORIENTATIONS OF GRAPHS \\ BY
}

\author{
CURTIS GREENE AND THOMAS ZASLAVSKY
}

\begin{abstract}
The doubly indexed Whitney numbers of a finite, ranked partially ordered set $L$ are (the first kind) $w_{i j}=\Sigma\left\{\mu\left(x^{i}, x^{j}\right): x^{i}, x^{j} \in L\right.$ with ranks $\left.i, j\right\}$ and (the second kind) $W_{i j}=$ the number of $\left(x^{i}, x^{j}\right)$ with $x^{i} \leqslant x^{j}$. When $L$ has a 0 element, the ordinary (simply indexed) Whitney numbers are $w_{j}=w_{0 j}$ and $W_{j}=W_{0 j}$ $=W_{j j}$. Building on work of Stanley and Zaslavsky we show how to interpret the magnitudes of Whitney numbers of geometric lattices and semilattices arising in geometry and graph theory. For example: The number of regions, or of $k$-dimensional faces for any $k$, of an arrangement of hyperplanes in real projective or affine space, that do not meet an arbitrary hyperplane in general position. The number of vertices of a zonotope $P$ inside the visible boundary as seen from a distant point on a generating line of $P$. The number of non-Radon partitions of a Euclidean point set not due to a separating hyperplane through a fixed point. The number of acyclic orientations of a graph (Stanley's theorem, with a new, geometrical proof); the number with a fixed unique source; the number whose set of increasing arcs (in a fixed ordering of the vertices) has exactly $q$ sources (generalizing Rényi's enumeration of permutations with $q$ "outstanding" elements). The number of totally cyclic orientations of a plane graph in which there is no clockwise directed cycle. The number of acyclic orientations of a signed graph satisfying conditions analogous to an unsigned graph's having a unique source.
\end{abstract}

Introduction. Wherever there is a finite matroid or geometric lattice, there are Whitney numbers. This article concerns their enumerative interpretation.

The coefficients of the chromatic polynomial of a graph, for one example, are Whitney numbers of the graphic matroid. Stanley found for the sum of their magnitudes an unconventional interpretation: it is the number of acyclic orientations [23]. The Whitney numbers in question are those of the first kind; for a finite matroid $M$ they are defined as the coefficients of its characteristic polynomial, thus

$$
w_{j}(M)=\sum\{\mu(\varnothing, x): x \text { closed in } M, r(x)=j\} .
$$

(Here $\mu$ denotes the Möbius function of $M$.)

Received by the editors March 30, 1982.

1980 Mathematics Subject Classification. Primary 05B35; Secondary 05C20, 05C38, 05C99, 51M20, 52A25, 52A35.

Key words and phrases. Matroid, combinatorial geometry, geometric lattice, Whitney numbers, arrangement of hyperplanes, partition of space, zonotope, Radon partition, digraph, oriented graph, acyclic orientation, totally cyclic orientation, signed graph. 
Another example: Let $M$ be the linear dependence matroid of a set $\mathcal{H}$ of hyperplanes arranged in Euclidean or projective space. The sum of the magnitudes $w_{j}^{+}$is the number of regions ( $d$-dimensional cells) into which $\mathcal{H}$ dissects the space (times $\frac{1}{2}$ if projective) [24]. Dualizing, let $M$ be the affine dependence matroid of a finite set $S$ in Euclidean space. Then $\sum w_{j}^{+}$is the number of non-Radon bipartitions of $S$ : partitions into the two sides of some affine hyperplane [24].

Still another example: Let $M$ be a regular ("unimodular") matroid. The sum of the magnitudes $w_{j}^{+}$is the number of row equivalence classes of totally unimodular representation matrices for $M$ in which no minimal dependent set of columns sums to zero [7, Proposition 4.5].

A generalization that includes all the examples: The sum is the number of acyclic orientations of an oriented matroid (defined in [4]) $[15,18]$. It is also the number of regions of an arrangement of topological hyperplanes; one can see this by combining with Las Vergnas' oriented matroid enumerations the correspondence between oriented matroids and arrangements of topological hyperplanes [12, §IV], or alternatively, independently of oriented matroids, by [26, §3] (see also [25, §2]).

Another sum of Whitney numbers is the beta invariant

$$
\beta(M)=(-1)^{r(M)} \sum_{j} j w_{j}(M)
$$

of Crapo [10]. This for a projective arrangement of hyperplanes equals the number of regions not touching a particular one of the hyperplanes [24]. This interpretation carries over exactly to oriented matroids, although the statement has apparently not appeared in the literature. It extends as well to arrangements of topological hyperplanes, either through their correspondence with oriented matroids, or by [26, §3], although no topologically complete proof on the latter line has yet been formulated (see the remarks in [26, p. 276]). Considering the hyperplane arrangement representing a graph we are led to interpret $\beta$ as the number of acyclic orientations in which a fixed node $p$ is the only source and a fixed adjacent node $q$ is the only sink. Alternatively $\beta$ is the number of acyclic orientations that become totally cyclic when a fixed edge $e$ is reversed-an interpretation that generalizes to oriented matroids $[16,17]$. Berman has independently obtained the same result for planar graphs through another approach based on internal and external activities [3], extended (with modifications) to oriented matroids by Las Vergnas [17, §3].

But the terms in these sums-the Whitney numbers themselves-have not been interpreted. It is our main purpose to show how to attach to each Whitney number magnitude $w_{j}^{+}$a geometric or graphical meaning in the several contexts of the title. We emphasize geometrical thinking and obtain as many results as we can, including interpretations of basepointed and doubly indexed Whitney numbers of the first kind, the various sums, and also the Whitney numbers of the second kind $W_{j}(M)$, the number of rank $j$ flats, whose meaning is relatively evident. We interpret the dual Whitney numbers of a graph in terms of totally cyclic orientations, and some of the Whitney invariants of a signed graph. The proofs are short because the facts are, on the whole, simple; what we contribute is for the most part the right viewpoint, the explicit statement, and the telling instance. 
1. Abstract preparation. Whitney numbers can be defined on any ranked partially ordered set. ${ }^{1}$ The rank function on $P$ we denote by $r$; the lowest rank is 0 ; the rank of $P$ is $r(P)=\max \{r(x): x \in P\}$. Writing $x^{i}, y^{i}$ for elements of $P$ of rank $i$ and $P^{i}$ for the set of all such elements, we define the doubly indexed Whitney numbers of the first kind,

$$
w_{i j}(P)=\sum_{x^{\prime} . x^{\prime} \in P} \mu\left(x^{i}, x^{j}\right)
$$

and of the second kind,

$$
W_{i j}(P)=\sum_{x^{i}, x^{\prime} \in P} \zeta\left(x^{i}, x^{j}\right)=\#\left\{\left(x^{i}, x^{j}\right): x^{i} \leqslant x^{j}\right\} .
$$

The usual Whitney numbers are the simply indexed ones:

$$
w_{j}(P)=w_{0 j}(P), \quad W_{j}(P)=W_{0 j}(P) .
$$

If $P$ has 0 and 1, we define the Möbius invariant $\mu(P)=\mu(0,1)=w_{r(P)}(P)$.

The partially ordered sets of interest to us are first of all $L(M)$, the geometric lattice of closed sets in a matroid $M$, and secondly for any point $b$ of $M$ the semilattice

$$
L(M, b)=\{x \in L(M): b \notin x\},
$$

the semilattice of the basepointed matroid $(M, b)$. (We shall assume throughout that $\varnothing$ is closed in all matroids; otherwise some special definitions are necessary.)

The beta invariant of $L(M)$ was defined in the introduction. An extremely useful fact from [24, Proof of Theorem D] is that for any matroid of rank $r \geqslant 1$ we have

$$
\beta(L(M))=(-1)^{r-1} \sum_{j=0}^{r-1} w_{j}(L(M, b)) .
$$

Another important fact is that the value of $w_{j}(L(M, b))$ is independent of $b$. This is a consequence of Weisner's Theorem [22, p. 351], which implies that

$$
w_{j}^{+}(L(M, b))=w_{j+1}^{+}(L(M))-w_{j+1}^{+}(L(M, b)) .
$$

Thus we may write

$$
w_{j}^{\prime \prime}(L(M))=\text { the common value of } w_{j}(L(M, b)) \text { for all } b \text { in } M .
$$

Consequently,

$$
w_{j}^{+}(L(M))=w_{j-1}^{\prime \prime}(L(M))+w_{j}^{\prime \prime+}(L(M)) .
$$

On the other hand the quantities $w_{i j}(L(M, b))$ for $i>0$ are not in general independent of $b$.

Some more notation: the contraction $P / x$ is $\{y \in P: y \geqslant x\}$. The interval $[x, z]$ is $\{y \in P / x: y \leqslant z\}$. The poset truncation operator $T$ applied to a ranked poset removes the top rank; the lattice truncation operator $\hat{T}$ removes the elements covered by 1 in a lattice.

\footnotetext{
'In this paper all posets, sets of hyperplanes or points, graphs, etc., are finite.
} 
2. Fundamentals of arrangements. A Euclidean arrangement of hyperplanes is a set $\delta$ of hyperplanes in the real affine space $\mathbf{E}^{d}$ together with the associated dissection of the space into cells of various dimensions. We consider the cells to be relatively open, so they have no points in common. For the number of $k$-cells ( $k$-dimensional cells) we write $f_{k}(\varepsilon)$.

A flat (of $\mathcal{E}$ ) is a nonvoid intersection of members of $\mathcal{E}$ (including $\mathbf{E}^{d}=\cap \varnothing$ ). We write $L(\mathcal{E})$ for the set of all flats ordered by reverse inclusion and $a_{k}(\mathcal{E})$ for the number of $k$-flats ( $k$-dimensional flats). The set $L(\mathcal{E})$ is a ranked poset having rank function

$$
r(x)=d-\operatorname{dim} x
$$

and having total rank

$$
r(\mathcal{E})=r(L(\mathcal{E}))=d-\min _{x \in I .(\mathcal{E})} \operatorname{dim} x
$$

it has a zero $\left(0=\mathbf{E}^{d}\right)$, and it has Whitney numbers. It is a geometric lattice if (and only if) $\mathcal{E}$ is central, that is $\cap \mathcal{E} \neq \varnothing$; in general it is the semilattice of a basepointed matroid (see below).

Clearly

$$
a_{k}(\mathcal{E})=W_{d-k}(L(\mathcal{E}))
$$

A more subtle link between arrangements and semilattices is

THEOREM A [24]. For a Euclidean arrangement of hyperplanes $\mathcal{E}$ in $\mathbf{E}^{d}$ we have

$$
f_{k}(\mathcal{E})=\sum_{j=d-k}^{r(\mathcal{E})} w_{d-k, j}^{+}(L(\mathcal{E})) .
$$

If $r(\mathcal{E})=d$, then $\mathcal{E}$ has vertices; thus it has bounded cells and possibly bounded regions. Although $\mathcal{E}$ cannot have any bounded cells if $r(\mathcal{E})<d$, it is true that all flats of maximum rank $r(\mathcal{E})$ (relative vertices) are translates of each other [24, Lemma 2D1]; if we section $\mathcal{E}$ by any affine subspace $s$ of dimension $r(\mathcal{E})$, transverse to the relative vertices, we get an arrangement $\mathcal{E}_{s}$ in $s$ combinatorially isomorphic to $\mathcal{E}$ but with all dimensions reduced by $d-r(\mathcal{E})$. A cell of $\mathcal{E}$ that becomes bounded in $\mathcal{E}_{s}$ we call relatively bounded. Let $b_{k}(\mathcal{E})$ be the number of relatively bounded $k$-cells.

THEOREM C [24]. For a Euclidean arrangement $\mathcal{E}$ in $\mathbf{E}^{d}$ we have

$$
b_{k}(\mathcal{E})=(-1)^{r(\mathcal{E})} \sum_{j=d-k}^{r(\mathcal{E})} w_{d-k, j}(L(\mathcal{E})) .
$$

Suppose we write $\hat{L}(\mathscr{E})$ for $L(\mathscr{E}) \cup\{\hat{1}\}$, where $\hat{\imath}$ is a special element added on top. Then $\hat{L}(\mathcal{E})$ is a ranked lattice (not usually geometric) with rank $r(\mathcal{E})+1$. Let $r=r(\mathcal{E})$ for brevity. Theorem $\mathrm{C}$ can be restated as

$$
b_{k}(\varepsilon)=(-1)^{r+1} w_{d-k, r+1}(\hat{L}(\mathcal{E}))
$$

(this is [24, Corollary 2.2]).

On any affine subspace $s, \mathcal{E}$ induces an arrangement

$$
\varepsilon_{s}=\{h \cap s: h \in \mathcal{E}, h \nsupseteq s, h \cap s \neq \varnothing\} .
$$


The restriction of $\mathcal{E}$ over $s$ is the subarrangement

$$
\mathcal{E}(s)=\{h \in \mathcal{E}: h \supseteq s\} ;
$$

it is central if $s \neq \varnothing$. If $x$ is a flat, then

$$
L\left(\varepsilon_{x}\right)=L(\mathcal{E}) / x \text { and } L(\mathcal{E}(x))=[0, x]_{L(\mathcal{E})} .
$$

If at the other extreme $g$ is a subspace in (relatively) general position with respect to $\mathcal{E}$, meaning that it is parallel to the relative vertices and meets each flat in the smallest possible dimension (hence it meets precisely the flats $x$ satisfying $\operatorname{dim} x \geqslant$ $r(\mathcal{E})-\operatorname{dim} g$, and then $\operatorname{dim}(g \cap x)=\operatorname{dim} g-r(x))$, then

$$
L\left(\varepsilon_{g}\right)=T^{d-\operatorname{dim} g} L(\varepsilon) \text {. }
$$

A projective arrangement of hyperplanes is a nonvoid set $Q$ of hyperplanes in the real projective space $\mathbf{P}^{d}$, together with the associated dissection of $\mathbf{P}^{d}$. The notations $a_{k}(Q)$ and $f_{k}(Q)$ are as before, but $\varnothing$ is not excluded as a flat or cell: a flat is any intersection of hyperplanes, so $L(\mathbb{Q})$ is always a geometric lattice with rank function as before. We have thus

$$
a_{k}(\mathbb{Q})=W_{d-k}(L(Q))
$$

and the theorem:

THEOREM B [24]. For a projective arrangement of hyperplanes in $\mathbf{P}^{d}$ and for $k>d-r(\mathbb{Q})$ we have

$$
f_{k}(\mathbb{Q})=\frac{1}{2} \sum_{j=d-k}^{r(\mathbb{Q})} w_{d-k, j}^{+}(L(\mathbb{Q})),
$$

and we have $f_{d-r(\mathbb{Q})}(\mathscr{Q})=1$.

This result follows from Theorem $A$ by regarding $Q$ as the image of a central arrangement $\hat{\mathscr{Q}}$ in $\mathbf{E}^{d+1}$; each opposite pair of $k+1$-cells of $\hat{\mathscr{Q}}$ becomes a $k$-cell of $\mathcal{Q}$, except for the smallest cell $\cap \hat{\mathbb{Q}}$. The lattices $L(\mathscr{Q})$ and $L(\hat{\mathbb{Q}})$ are canonically isomorphic, whence their Whitney numbers are the same. What this construction means in general is that one can deduce results about $\mathcal{Q}$ from Euclidean arguments about $\hat{\mathbb{C}}$.

In the other direction, any Euclidean arrangement $\mathcal{E}$ in $\mathbf{E}^{d}$ has a projectivization: the arrangement $\mathcal{E}_{\mathbf{P}}$ in $\mathbf{P}^{d}$ obtained by adjoining to $\mathcal{E}$ the ideal hyperplane $\infty$. Evidently $\mathcal{E}_{\mathbf{P}}$ and $\mathscr{E}$ have the same number of regions. What is more surprising is that the bounded regions of $\mathcal{E}$ can be counted in $\mathcal{E}_{\mathbf{P}}$.

THEOREM D [24]. A Euclidean arrangement $\mathcal{E}$ has $\beta\left(L\left(\mathcal{E}_{\mathbf{p}}\right)\right)$ relatively bounded regions.

Conversely we call an affinization of a projective arrangement $\mathbb{Q}$ any Euclidean arrangement obtained by regarding one of the hyperplanes in $Q$ as the infinite hyperplane. The semilattice of the affinization by $h$ we denote by $L(Q, h)$.

A projective arrangement $Q$ induces an arrangement $\mathbb{Q}_{s}$ and determines a restriction $\mathbb{Q}(s)$ for any projective subspace $s$; and for $x \in L(\mathbb{Q})$ the lattices of these 
arrangements are as with Euclidean arrangements. If $g$ is a subspace in (relatively) general position with respect to $\mathcal{Q}$, meaning that $g \supseteq \cap \mathbb{Q}$ and $g$ meets each flat $x$ in the smallest possible dimension (which is $\max [\operatorname{dim} g-r(x), \operatorname{dim} \cap \mathbb{Q}]$ ), we have

$$
L\left(Q_{g}\right)=\hat{T}^{d-\operatorname{dim} g} L(Q) .
$$

It is worth noting that all results about cells in projective arrangements extend to oriented matroids and all Euclidean results extend to basepointed oriented matroids. (Thus they apply to arrangements of topological hyperplanes.) The possibility of such extension is made clear by Las Vergnas' development of the enumeration theory of reorientations of an oriented matroid [15-18]. The extensions of most of our results have not been worked out precisely; the task is straightforward, but since the details tend to become quite technical, we restrict ourselves here to "real" situations.

3. Euclidean arrangements. We begin by interpreting in two ways the "upper" Whitney numbers $w_{i r}^{+}(L(\mathcal{E}))$ of a Euclidean arrangement $\varepsilon$. That allows us to interpret $\mu^{+}(0, x)$ for any flat $x \in L(\varepsilon)$ by applying the theorems to $\varepsilon(x)$, whose lattice is the interval $[0, x]$ in $L(E)$.

THEOREM 3.1. Let $\varepsilon$ be a Euclidean arrangement in $\mathbf{E}^{d}$ with rank $r$. Let $g$ be a general hyperplane with respect to $\xi$. Then $g$ meets all but exactly $w_{r}^{+}(L(\xi))$ regions of $\mathcal{E}$ and all but exactly $w_{d-k, r}^{+}(L(\varepsilon))$ of its $k$-cells.

Proof. Compare Theorem A for (5) and for $\varepsilon_{g}$ in the light of (2.3).

The second theorem requires \&) to be central.

THEOREM 3.2. Let $\mathcal{E}$ be a central arrangement in $\mathbf{E}^{d}$ with rank $r$. Let $g$ be a hyperplane general with respect to $\xi_{\text {. }}$. Then the induced arrangement $\xi_{g}$ has $\mu^{+}(0,1)=$ $w_{r}^{+}(L(\mathcal{E}))$ relatively bounded regions and $w_{d-k, r}^{+}(L(\mathcal{E}))$ relatively bounded $k-1$-cells.

Proof. Apply (2.3) to $\mathcal{E}_{\text {and }}(2.2)$ to $\mathcal{E}_{g}$.

COROLlaRY 3.1. Let $\mathcal{E}$ be a Euclidean arrangement with rank $d$ and let $k>0$. Also let $g$ be a hyperplane general with respect to \&) except for containing one or more vertices $v_{1}, \ldots, v_{q}$. If $g$ moves slightly parallel to itself, it meets besides all the $k$-cells it originally met exactly $\sum_{m=1}^{q} w_{d}^{+} k, d\left(L\left(\varepsilon\left(v_{m}\right)\right)\right)$ new ones.

Proof. The $k$-cells met by $g$ correspond to $k$ - 1-cells of $\varepsilon_{g}$. The $k$ - l-cells that appear as $g$ moves away from $v_{m}$ are those bounded hy hyperplanes through $v_{m}$. So apply Theorem 3.2. That suffices if $q=1$; otherwise we must know there is no multiple counting. But if there were, a multiply counted $k$-cell would have had (say) both $v_{m}$ and $v_{l}$ as vertices and the segment between them as an edge; thus $g$ would have contained a line of $\mathcal{E}$, contrary to generality.

COROLlaRY 3.2. Let $\mathcal{E}$ be a Euclidean arrangement of rank $d$. Let $g_{d}=\mathbf{E}^{d} \supset g_{d-1}$ $\supset \cdots \supset g_{0} \supset g_{-1}=\varnothing$ be a chain of affine subspaces of the indicated dimensions, general with respect to $\mathcal{E}$. Then for each $l=d, d-1, \ldots, 0$, the number of $k$-cells of $\mathcal{E}$ that meet $g_{l}$ but not $g_{l-1}$ is equal to $w_{d-k, l}^{+}(L(\mathcal{E}))$. 
Proof. Apply (2.3) to get $L\left(\mathcal{E}_{g_{1}}\right)$. Then apply Theorem 3.1 to $\mathcal{E}_{g_{i}}$.

COROLlary 3.3. Let $\mathcal{E}$ and $g_{l}$ be as in Corollary 3.2. The number of $k$-cells of $\varepsilon$ met by $g_{l}$ is $\sum_{j \leqslant l} w_{d-k, j}^{+}(L(\mathcal{E}))$; the number missed by $g_{l}$ is $\sum_{j>l} w_{d-k, j}^{+}(L(\mathscr{E}))$.

The preceding results can serve as the basis for generalizing to higher dimensions the method of sweep lines and sweep planes exploited so successfully by Wetzel and his associates in $[1, \S 3 ; \mathbf{1 4} ; 2, \S 4]$, etc. We plan to treat this topic fully elsewhere, but the next theorem and the perturbation method of Corollary 3.1 suggest the approach.

In this theorem we carry further the term-by-term analysis of equations like $f_{d}(\varepsilon)=\Sigma_{l} w_{l}^{+}(L(\varepsilon))$, as in Corollary 3.2. We show how to break up the regions counted by each $w_{l}^{+}(L(\varepsilon))$ into blocks of sizes $\mu^{+}\left(0, x^{d}\right)$ for vertices $x^{d}$; and similarly for cells of other dimensions.

THEOREM 3.3. Let $\mathcal{E}$ be an arrangement of rank $d$ in $\mathbf{E}^{d}$. Take a hyperplane $g_{0}$, general with respect to $\mathcal{E}$, such that all the vertices of $\delta$ lie on one side of $g_{0}$ and no two vertices are equidistant from it, and number the vertices $v_{1}, v_{2}, \ldots, v_{q}$ in order of distance from $g_{0}$. For $1 \leqslant m<q$ let $g_{m}$ be a translate of $g_{0}$ lying between $v_{m}$ and $v_{m+1}$, and let $g_{q}$ be a translate of $g_{0}$ lying beyond $v_{q}$ (so $v_{1}, \ldots, v_{m}$ are the vertices between $g_{0}$ and $\left.g_{m}\right)$. Then for $m=1,2, \ldots, q, \mu^{+}\left(0, v_{m}\right)$ is the number of regions and $w_{d-k . d}^{+}$ $\left(\left[0, v_{m}\right]\right)$ is the number of $k$-cells met by $g_{m}$ but not by $g_{m-1}$.

Proof. When $\&$ is central, $q=1$ and the theorem is true by Theorem 3.1: for $g_{0}$ and $g_{1}$ between them meet every $k$-cell.

In general the cells in question, say $C_{1}, \ldots, C_{j}$, are just those that have $v_{m}$ as a vertex and have no edge meeting $g_{m-1}$. If we discard all hyperplanes not in $\mathscr{E}\left(v_{m}\right)$, then $C_{1}, \ldots, C_{j}$ become enlarged to cones at $v_{m}$; but they are still distinct and do not meet $g_{m-1}$. Moreover all other $k$-cells of $\varepsilon\left(v_{m}\right)$ do meet $g_{m-1}$. So we apply the central case to complete the proof.

This theorem interprets $\mu^{+}(0, x)$ for $x \in L(\mathscr{E})$ as follows: Fix a chain of subspaces as in Corollary 3.2 , but chosen so in each $\mathcal{E}_{g_{l}}$ the relative hyperplane $g_{l-1,0}=g_{l-1}$ satisfies the criteria of Theorem 3.3. Then apply Theorem 3.3 to $\varepsilon_{g_{l}}$ with $g_{l-1,0}=g_{l-1}$ and with $g_{l-1,1}, \ldots, g_{l-1, q}$ suitable translates.

Thus we have interpreted the Whitney numbers of the first kind. The interpretation of the second kind is relatively trivial (cf. (2.1)), but it is curious that Corollary 3.2 for regions $(k=d)$ has an exact analog for flats obtained by changing $w_{l}^{+}(L(\mathcal{E}))$ to $W_{l}(L(\mathbb{E}))$. One wonders whether there is an interesting extension to the doubly indexed numbers $W_{i l}(L(\varepsilon))$.

In Corollary 3.1 we perturbed a fairly general hyperplane through $v$. Now suppose we shift one of the hyperplanes of a central arrangement like $\mathcal{E}(v)$.

THEOREM 3.4. Let $\mathcal{H}$ be a central arrangement in $\mathbf{E}^{d}$ and let $h \in \mathcal{H}$. Let $h^{*}$ be $h$ perturbed by translation from its initial position and let $\mathcal{H}^{*}$ be the perturbed arrangement, with or without $h$ also. Then

$$
b_{d}\left(\mathcal{H}^{*}\right)=b_{d-1}\left(\mathcal{H}_{h^{*}}\right)=\beta(L(\mathcal{H})),
$$

regardless of the choice of $h$. 
Proof. The idea is to go over to $\mathbf{P}^{d-1}$, in which we regard $h$ as the ideal hyperplane. Removing $h$ leaves a Euclidean arrangement, isomorphic to $\mathcal{H}_{h^{*}}$, to which Theorem D can be applied. Since $\mathcal{H}^{*}$ (without $h$ ) is a cone over $\mathcal{H}_{h^{*}}$, and $h$ introduces no more bounded regions, the rest follows.

COROLlaRy 3.4. With notation as in Theorem 3.4, let also $r=r(\mathcal{H})$ and $d-r<k$ $\leqslant d$. Then

$$
b_{k}\left(\mathcal{H}_{h^{*}}\right)=(-1)^{r-(d-k)} \sum_{j=d-1-k}^{r-1} w_{d-1-k, j}^{+}(L(\mathcal{H}, h))
$$

and

$$
b_{k}\left(\mathcal{H}^{*}\right)=b_{k}\left(\mathcal{H}_{h^{*}}\right)+b_{k-1}\left(\mathcal{H}_{h^{*}}\right) \text {. }
$$

Proof. The corollary is valid for regions by Theorem 3.4 and (1.1).

For $k<d$ every relatively bounded $k$-cell is a region of some $k$-dimensional flat $x \in L\left(\mathcal{H}^{*}\right)$. If $x \geqslant h^{*}$, the cell is a relatively bounded $k$-cell of $\mathcal{H}_{h^{*}}$. If $x \geqslant h$, there can be no relatively bounded $k$-cells in $x$. The remaining case is that in which $y=h^{*} \vee x$ plays the role of $h^{*}$ in the arrangement $\mathcal{H}_{x}^{*}$; thus by Theorem 3.4 we have $b_{k}\left(\mathcal{H}_{x}^{*}\right)=b_{k-1}\left(\mathcal{H}_{y}\right)$. From this we conclude the second formula of the corollary.

Also by Theorem 3.4, since any $y \in L\left(\mathcal{K}_{h^{*}}\right)^{d-1-k}$ has the form $x \vee h^{*}$ for a unique $x \in L(\mathcal{K}, h)^{d-1-k}$, we have

$$
b_{k}\left(\mathcal{H}_{y}\right)=b_{k+1}\left(\mathcal{H}_{x}\right)=(-1)^{r-d+k} \sum_{j=d-1-k}^{r-1} w_{j-(d-1-k)}^{+}(L(\mathcal{K}, h) / x) .
$$

Summing over all $y$, equivalently over all $x$, gives the first formula of the corollary.

In the extreme case $k=d-r$ we have

$$
b_{d-r}\left(\mathcal{H}^{*}\right)=b_{d-r}(\mathcal{H})+1=W_{r-1}(L(\mathcal{H}))+1
$$

provided $\mathcal{H}^{*}$ contains $h$, or $h$ is not an isthmus of $L(\mathcal{H})$.

Suppose $\mathcal{E}$ is a central arrangement with center $z=\cap \mathcal{E}$ and $g$ is a general hyperplane (parallel to $z$, recall). Then we can count bounded regions of $\mathcal{E} \cup\{g\}$ in two ways: by Theorem 3.1, or by taking $h$ to be the translate of $g$ passing through $z$ and applying Theorem 3.4 to $\mathcal{E} \cup\{h\}$. Comparing the two answers yields a geometrical proof of the identity

$$
\mu^{+}(L(\mathcal{E}))=\beta(L(\mathcal{E} \cup\{h\})) .
$$

The algebraic proof is that, since $g$ is general, the matroid of $L(\mathcal{E} \cup\{h\})$ is the general rank-preserving one-point extension of that of $L(\mathcal{E})$. Thus $w_{j}(L(\mathcal{E}))=$ $w_{j}^{\prime \prime}(L(\mathcal{E} \cup\{h\}))$ for $j<r$ and we get (3.1) from (1.1).

We conclude by giving the generalization of Theorem 3.1 and Corollary 3.1 to arbitrary hyperplanes $g$. The proof this time is abstract, following the lines of $[\mathbf{2 4}, \mathrm{pp}$. 50-52]. 
THEOREM 3.5. Let $\mathcal{E}$ be a Euclidean arrangement in $\mathbf{E}^{d}$, let $g$ be a hyperplane in $\mathbf{E}^{d}$, and let

$$
M=\{t \in L(\mathcal{E}): t \subseteq g\}, \quad N_{0}=\{t \in L(\mathcal{E}): t \cap g=\varnothing\} .
$$

Then $g$ meets all but exactly $\Sigma_{t \in N_{0}} \mu^{+}(0, t)+2 \Sigma_{t \in M} \mu^{+}(0, t)$ regions of $\mathcal{E}$ and all but exactly

$$
\sum_{\substack{s \in L(\delta) \backslash M \\ \operatorname{dim} s=k}}\left\{\sum_{t \in N_{0}} \mu^{+}(s, t)+2 \sum_{t \in M} \mu^{+}(s, t)\right\}
$$

of its $k$-cells.

Proof. Let $L_{1}=L(\varepsilon \cup\{g\})$ and

$$
C=\{t \in L(\mathcal{E}) \backslash M: t \cap g \in L(\mathcal{E})\}, \quad N=L(\mathcal{E}) \backslash(M \cup C) .
$$

Thus $N_{0} \subseteq N$. The number of regions of $\mathscr{E}$ met by $g$ equals $f_{d-1}\left(\mathscr{E}_{g}\right)$, so the number missed is

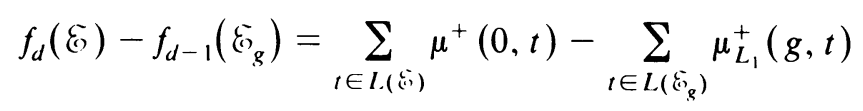

by Theorem A. Now $[0, t]_{l \text { (泣) }} \cong[g, t \vee g]_{l_{1}}$ for $t \in N \backslash N_{0}$, and $L_{1} / g=L\left(\mathcal{E}_{g}\right)=$ $M \cup\left\{t \vee g: t \in N \backslash N_{0}\right\}$. Also

$$
\sum_{t \in C} \mu^{+}(0, t)=\sum_{t \in M} \mu_{l_{1}}^{+}(0, t)=\sum_{t \in M}\left[\mu^{+}(0, t)+\mu_{L_{1}}^{+}(g, t)\right]
$$

by Corollary (a) to Proposition 4 in $[22, \S 5]$ (as explained in [24, Corollary 4C5]) and the deletion-contraction law $\mu_{L_{1}}^{+}(0, t)=\mu_{L_{1}}^{+}(g, t)+\mu^{+}(0, t)$ (cf. [5] for instance). Thus (*) equals the desired expression.

The number of $k$-cells missed is obtained by summing the number in each $k$-flat $s \not g$.

If $\mathcal{E}$ is central and $g$ does not contain the center, Theorem 3.5 simplifies, for then $M=\varnothing$. If $g$ does contain the center, then contrariwise $N_{0}=\varnothing$.

4. Projective arrangements. We start by observing that Corollary 3.1 has an analog for projective arrangements. This interprets $\mu^{+}(0, v)$ if we take $k=d, q=1$, and $p=0$.

COROllaRy 4.1. Let $Q$ ' be a projective arrangement in $\mathbf{P}^{d}$ with rank $d+1$ and let $k>0$. Let $g$ be a hyperplane that contains the vertices $v_{1}, \ldots, v_{q}$ but no line of $Q$. If $g$ is shifted slightly so it contains only $v_{1}, \ldots, v_{p}$ (where $p$ may be 0$)$, then it meets, besides all the original $k$-cells, exactly $\sum_{m=p+1}^{q} w_{d-k, d}^{+}\left(L\left(\mathscr{Q}\left(v_{m}\right)\right)\right)$ new ones.

Proof. We need consider only the case $p=0$; as in the proof of Corollary 3.1 it suffices to treat the case $q=1$. Since only local effects matter if $g$ is moved but slightly, we can throw to infinity a distant hyperplane of $\mathbf{P}^{d}$ (not necessarily in $\mathbb{Q}$ ) and in the resulting affine space appeal to Corollary 3.1.

Now we interpret the $w_{i r}^{+}(L(\mathbb{Q}))$. 
THEOREM 4.1. Let $Q$ be a projective arrangement of rank $r$ and let $k>d-r$. Let also $g$ be a general hyperplane with respect to $Q$. Then $g$ meets all but exactly $w_{d-k, r}^{+}(L(Q))$ of the $k$-cells of $Q$.

Proof. The number of $k$-cells $g$ meets is the number of $k-1$-cells of $Q_{g}$. Since $g$ is general, whence $L\left(Q_{g}\right)=\hat{T} L(\mathbb{Q})$, that number is

$$
f_{k-1}\left(Q_{g}\right)=\frac{1}{2}\left\{\sum_{j=d-k}^{r-2} w_{d-k, j}^{+}(\hat{T} L(Q))+w_{d-k, r-1}^{+}(\hat{T} L(Q))\right\} .
$$

Now $w_{i j}(\hat{T} L)=w_{i j}(L)$ if $j<r-1$ and $w_{i, r-1}(\hat{T} L)=w_{i r}(L)+w_{i, r-1}(L)$ if $i \leqslant r-$ 1. By Rota's sign theorem [22] we deduce

$$
f_{k-1}\left(Q_{g}\right)=\frac{1}{2} \sum_{j=d-k}^{r} w_{d-k, j}^{+}(L(Q))-w_{d-k, r}^{+}(L(Q)),
$$

whence $g$ misses exactly $w_{d-k, r}^{+}(L(\mathbb{Q}))$ of the $k$-cells.

COROLlaRY 4.2. Let $Q$ be a projective arrangement of rank $d+1$ and let $0 \leqslant k \leqslant d$. Let $g_{d}=\mathbf{P}^{d} \supset g_{d-1} \supset \cdots \supset g_{0} \supset g_{-1}=\varnothing$ be a chain of subspaces of the indicated dimensions, general with respect to $Q$. Then for each $l=d, \ldots, 1,0$, the number of $k$-cells of Q that meet $g_{l}$ but not $g_{l-1}$ is equal to

$$
\sum_{x^{d-k} \in I(\mathbb{Q})} w_{l-d-k}^{\prime \prime+}\left(L(\mathbb{Q}) / x^{d-k}\right) .
$$

Proof. Let us rewrite Theorem 4.1 by (1.2): the quantity there is

$$
\sum_{x^{d-k} \in I(Q)} w_{k+1}^{+}\left(L(Q) / x^{d-k}\right)=\sum_{x^{d}{ }_{k} \in I\left(Q^{*}\right)} w_{k}^{\prime \prime+}\left(L(Q) / x^{d-k}\right) .
$$

The $k$-cells of $Q$ that meet $g_{\text {l }}$ correspond exactly to the $k-(d-l)$-cells of $\mathbb{Q}_{g_{i}}$. The lattice of the latter is $\hat{T}^{d-1}(L(\mathcal{Q}))$, whose rank is $l+1$. So by Theorem 4.1 the number we seek is

$$
\sum\left\{w_{k-d+l, l+1}^{\prime \prime}\left(\hat{T}^{d-l}(L(Q)) / x^{d-k}\right): x^{d-k} \in \hat{T}^{d-l}(L(\Theta))\right\}
$$

provided $d-k<l+1$. Then the range of $x^{d-k}$ is $L(\mathcal{Q})^{d-k}$. Furthermore $k-d+$ $1<l+1$, so the summand simplifies to $w_{k-d+l, l+1}^{\prime \prime}\left(L(Q) / x^{d-k}\right)$, as desired.

But if $d-k \geqslant l+1$, the summand is identically 0 , as required for the corollary.

There is, of course, a refinement of Corollary 4.2 analogous to Theorem 3.3.

COROllary 4.3. Let $\mathcal{Q}, k$, and $g_{l}$ be as in Corollary 4.2. Then (with congruences modulo 2) the number of $k$-cells met by $g_{l}$ is

$$
\sum_{j \leqslant l, j \equiv l} w_{d-k, j}^{+}(L(Q))
$$

and the number missed by $g_{l}$ is

$$
\sum_{j>l, j \equiv l} w_{d-k, j}^{+}(L(\mathbb{Q})) .
$$


Proof. A matter of summing as suggested by the previous corollary and simplifying by means of (1.2).

The Whitney numbers of the second kind interpret by an analog of Corollary 4.2 as with Euclidean arrangements.

5. Zonotopes. A zonotope is the vector sum of a finite number of closed line segments in the real linear space $\mathbf{R}^{d}$; it is a convex polytope. We may suppose without loss of generality that all the segments $S_{1}, \ldots, S_{n}$ are centered on the origin; then $S_{m}$ is the convex hull of its endpoints $z_{m}$ and $-z_{m}$. We write $Z=\left\{z_{1}, \ldots, z_{n}\right\}$, and $P(Z)=S_{1}+\cdots+S_{n}$ for the zonotope. We assume for simplicity that all segments are nonzero and nonparallel. We write $f_{i}(P(Z))$ for the number of $i$-faces (that is, $i$-dimensional faces) of $P(Z)$, including $P(Z)$ but not $\varnothing$; the faces are considered to be relatively open. The zone of a segment $S_{m}$ is the union of all faces parallel to it.

We write $\mathbf{R}^{d}$ for the ambient space to stress that all subspaces are linear: they pass through the origin. Let $F$ be a face. The apex of $F$ is the linear space parallel to it: that is, it is aff $F$ translated to go through 0 . The (closed) cone of $F$ is obtained by first translating $P(Z)$ to $P(Z)_{F}$, in which 0 lies in the translate of $F$; then

$$
\text { cone }(F)=\left[\operatorname{pos} P(Z)_{F}\right] \cup\left[-\operatorname{pos} P(Z)_{F}\right] \text {, }
$$

where pos means positive span. Thus apex $(F)$ is in a sense the apex of cone $(F)$. We also define the open cone:

$$
\operatorname{cone}^{o}(F)=\operatorname{relint}\left[\operatorname{pos} P(Z)_{F}\right] \cup \operatorname{relint}\left[-\operatorname{pos} P(Z)_{F}\right] \cup\{0\} .
$$

The lattice of flats of $P(Z)$ is the set $L(Z)$ of linear subspaces spanned by $Z$, ordered by inclusion. It is a geometric lattice of $\operatorname{rank} r=\operatorname{dim} Z$ and is the lattice of the linear dependence matroid of $Z$. It also has the following interpretation: For a face $F$ let

$$
Z(F)=\left\{z_{m} \in Z: S_{m} \| F\right\}
$$

we take this to mean $Z(V)=\varnothing$ for a vertex $V$ and $Z(P(Z))=Z$. Then $P(Z(F))$ is a translate of $F$; and furthermore,

$$
L(Z)=\{\operatorname{lin} Z(F): F \text { is a face of } P(Z)\} .
$$

So the Whitney numbers of the second kind $W_{j}(L(Z))$, the number of $j$-dimensional subspaces spanned by $Z$, have an interpretation in $P(Z)$.

THEOREM 5.1. Let $P(Z)$ be a zonotope. The number of distinct sets of intersection of $j$ zones not the intersection of fewer zones is equal to $W_{j}(L(Z))$.

Each segment $S_{m}$ is dually a hyperplane $\hat{h}_{m}$; thus we have a central arrangement $\hat{\mathscr{Q}}$ in the dual space $\mathbf{R}^{d *}$ which carries over to a projective arrangement $Q=\left\{h_{1}, \ldots, h_{n}\right\}$ in $\mathbf{P}^{d-1}$. (Noted by Coxeter [9]. See also [19] for this duality and references.) The $i$-faces of $P(Z)$ correspond one-for-one to the $d-i$-cells of $\hat{\mathscr{Q}}$ and (when $i<r$ ) two-for-one to the $d-i-1$-cells of $Q$. Thus one gets enumerative properties of 
$P(Z)$ by dualizing those of arrangements. That is the topic of this section. First of course is

TheOrem 5.2 [24, CoRollary 6.3]. We have

$$
f_{i}(P(Z))=\sum_{j=i}^{r} w_{i j}^{+}(L(Z)) .
$$

A face is in the zone of $S_{m}$ iff it corresponds to a cell of the induced arrangement $Q_{h_{m}}$. Thus (when $i<r$ ) the number $f_{i}\left(P(Z), S_{m}\right)$ of $i$-faces not in the zone of $S_{m}$ is twice the number of $d-i-1$-cells in the affinization of $Q$ by $h_{m}$. By $L\left(Z, z_{m}\right)$ we denote the semilattice $L(Z) \backslash\left(L(Z) / z_{m}\right)$. From Theorem A we get

THEOREM 5.3. For $0 \leqslant i<r$ we have

$$
f_{i}\left(P(Z), S_{m}\right)=2 \sum_{j=i}^{r} w_{i j}^{+}\left(L\left(Z, z_{m}\right)\right) .
$$

Because the correspondence of faces to cells preserves incidence while reversing order, two closed faces intersect if and only if the corresponding cells of $\hat{\mathcal{Q}}$ intersect. Thus a closed face corresponds to a cell bounded in the affinization of $Q$ by $h_{m}$ iff it does not meet the closed zone of $S_{m}$. From Theorems D and C we deduce

THEOREM 5.4. If $n>0$, the number of opposite pairs of vertices of $P(Z)$ not in the closed zone of $S_{m}$ equals $\beta(L(Z))$. The number of opposite pairs of closed $i$-faces not meeting the closed zone of $S_{m}$ equals $\sum_{j} w_{i j}^{+}\left(L\left(Z, z_{i}\right)\right)$.

Another way to view Theorem 5.4 is this: Let $v$ be a point on lin $z_{m}$ and very far from $P(Z)$. Looking down from $v$ at $P(Z)$, we can see one vertex of each opposite pair. Those inside the visible part of $P(Z)$ are the ones not in the zone of $S_{m}$. So their number is $\beta(L(Z))$. Similarly the faces counted in Theorem 5.4 are the ones whose vertices are interior to the visible region of $P(Z)$.

A line $l$ in $\mathbf{R}^{d}$ corresponds to a hyperplane $h(l)$ in $\mathbf{P}^{d}$. The line is parallel to a facet iff $h(l)$ contains the corresponding vertex. Therefore what corresponds to a general hyperplane with respect to $\mathscr{Q}$ is a line parallel to no facet; we call this a general line with respect to $P(Z)$. A vertex whose closed (or, open) cone does not contain $l$ corresponds to a region (respectively, closed region) meeting $h(l)$. Thus an $i$-face $F_{i}$ of $P(Z)$ whose open cone does not contain $l$ corresponds to a closed $d-i-1$-cell of $Q$ meeting $h(l)$. An $F_{i}$ such that either $l \nsubseteq \not \operatorname{cone}\left(F_{i}\right)$ or $l \subseteq$ apex $\left(F_{i}\right)$ corresponds to a $d-i-1$-cell meeting $h(l)$. (Notice that any $l \subseteq \operatorname{apex}(F)$ is parallel to $F$.) We say that a subspace $t$ of $\mathbf{R}^{d}$ is external to $F_{i}$ if $t \cap \operatorname{cone}\left(F_{i}\right)=0$.

Now from Corollary 4.1 we have our first new result.

TheOREM 5.5. Let $P(Z)$ be a zonotope, where $Z$ spans $\mathbf{R}^{d}$, and let $0 \leqslant i<d$. Let l be a line that is parallel to facets $F^{(1)}, \ldots, F^{(q)}$ but not to any subfacet. Suppose $l$ is perturbed so it is parallel only to $F^{(1)}, \ldots, F^{(p)}$ (where $p$ may be 0 ) and to no subfacet. Then the number of $i$-faces $F_{i}$ to which $l$ is external is increased by

$$
2 \sum_{m=p+1}^{q} w_{i, r-1}^{+}\left(L\left(Z\left(F^{(m)}\right)\right)\right) .
$$


Remember that $L(Z(F))=[0, Z(F)]$ in $L(Z)$ for any face $F$. This gives an interpretation of $\mu^{+}(0, Z(F))$ for a facet $F_{r-1}$ by setting $i=0, q=1$, and $F^{(1)}=F$.

Corollary 5.1. Let $F$ be a facet of $P(Z)$, where $Z$ spans $\mathbf{R}^{d}$, and l a line parallel to $F$ but to no other proper face. Suppose $l$ is perturbed so it is parallel to no proper face. Then the number of vertices to which l is external is increased by $2 \mu^{+}(0, Z(F))$.

From Theorem 4.1 we can interpret $\mu^{+}(0,1)$ and the other "upper" Whitney numbers of $L(Z)$.

TheORem 5.6. Let $P(Z)$ be a zonotope and $i<r=\operatorname{dim} Z$. Let $l$ be a general line with respect to $P(Z)$. Then the number of opposite pairs of $i$-faces of $P(Z)$ to which $l$ is not external is exactly $w_{i r}^{+}(L(Z))$.

Another way of saying that a general line $l$ is not external to $F$ is to say that any translate of $l$ meeting $F$ also meets the interior of $P(Z)$.

Corollary 5.2. Let $P(Z)$ be a zonotope and $i<r=\operatorname{dim} Z$. Let $t_{0}=0 \subset t_{1} \subset$ $\cdots \subset t_{r}=\operatorname{lin} Z$ be a chain of subspaces of the indicated dimensions. Then the number of opposite pairs of $i$-faces of $P(Z)$ to which $t_{m-1}$, but not $t_{m}$, is external equals

$$
\sum_{x^{\prime} \in l .(Z)} w_{r-i-m}^{\prime \prime+}\left(L(Z) / x^{i}\right) \text {. }
$$

Corollary 5.3. Let $P(Z), i$, and $t_{m}$ be as in Corollary 5.2. With congruences modulo 2 , the number of opposite pairs of $i$-faces to which $t_{m}$ is external equals

$$
\sum_{j<r-m, j \not r-m} w_{i j}^{+}(L(Z)) .
$$

The number to which $t_{m}$ is not external equals

$$
\sum_{j>r-m, j \neq r-m} w_{i j}^{+}(L(Z)) .
$$

This corollary generalizes to lower-dimensional subspaces the observation that the translates of a general hyperplane support exactly two. opposite vertices and no higher faces.

There is an appealing interpretation of $\beta(L(Z)$ ) deduced from Theorem 3.4.

TheOREM 5.7. Let $P(Z)$ be a zonotope in $\mathbf{R}^{d}$ with $\operatorname{dim} Z<d$ and let $z_{m} \in Z$. Suppose the segment $S_{m}$ is rotated slightly towards the perpendicular to lin $Z$, into a new position $S_{m}^{*} \subseteq \operatorname{lin}\left(S_{m},(\operatorname{lin} Z)^{\perp}\right)$. Let $P\left(Z^{*}\right)$ be the zonotope generated by the perturbed segment set $\left\{S_{1}, \ldots, S_{m}^{*}, \ldots, S_{n}\right\}$ (optionally including $S_{m}$ as well), and let $v$ be a point on (lin $Z)^{\perp}$ far from $P(Z)$. Then the number of vertices visible from $v$ and interior to the visible part of $P\left(Z^{*}\right)$ is equal to $\beta(L(Z))$, regardless of the segment chosen to be rotated.

Proof. Without loss of generality we may assume lin $Z=\left\{x \in \mathbf{R}^{d}: x_{d}=0\right\}$. Let $a$ be the affine hyperplane $a=\left\{x \in \mathbf{R}^{d}: x_{d}=1\right\}$.

Dualizing $P(Z)$ and $P\left(Z^{*}\right)$, we get central arrangements $\hat{\mathscr{Q}}$ and $\hat{\mathbb{Q}}^{*}$ in $\mathbf{R}^{d}$. Then $\mathcal{H}=\hat{\mathbb{Q}}_{a}$ is a central Euclidean arrangement in $a$ with rank $d-1$, and $h\left(z_{m}^{*}\right) \cap a$ is a 
translate of $h\left(z_{m}\right) \cap a$. so $\mathfrak{H}^{*}=\hat{\mathfrak{Q}}_{a}^{*}$ is the perturbed arrangement in Theorem 3.4. That gives the number of bounded regions in $\hat{\mathfrak{Q}}_{a}^{*}$.

Let $z_{0}$ be a normal to lin $Z$ and $Z_{0}^{*}=Z^{*} \cup\left\{z_{0}\right\}$. The dual projective arrangement $\mathbb{Q}_{0}^{*}$ with $h\left(z_{0}\right)$ thrown to infinity is a Euclidean arrangement isomorphic to $\hat{\mathbb{Q}}_{a}^{*}$. Now the remarks preceding Theorem 5.4 allow us to interpret the bounded regions of $\hat{\mathbb{Q}}_{a}^{*}$ as the opposite pairs of vertices of $P\left(Z_{0}^{*}\right)$ not in the closed zone of $z_{0}$; by the comment after Theorem 5.4 we have the theorem.

There is a duality for zonotopes corresponding to matroid duality, under which a $d$-dimensional zonotope with $n$ zones corresponds to an $n-d$-dimensional zonotope

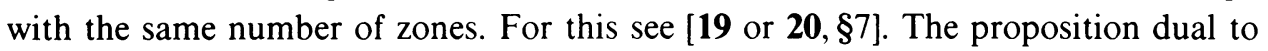
Theorem 5.2 with $i=0$ is

THEOREM 5.8. The number of points $\sum_{1}^{n} \varepsilon_{i} z_{i}$, where all $\varepsilon_{i}= \pm 1$, that lie in the interior of $P(Z)$ is equal to $\sum_{j} w_{j}^{+}\left(L^{\perp}(Z)\right)$, where $L^{\perp}(Z)$ is the lattice of the dual of the matroid of $Z$.

The proof is straightforward, given the zonotope duality theory in $[\mathbf{1 9}, \mathbf{2 0}]$.

6. Non-Radon partitions. A non-Radon partition of an affine set $S \subseteq \mathbf{E}^{d}$ is a partition of $S$ into the two parts on either side of a hyperplane not meeting $S$. (One such is the partition $\{\varnothing, S\}$.) A partition of $S$ into two parts not separable by a hyperplane is a Radon partition. A recent survey of the subject is [11].

One can dualize by regarding $S$ as a subset of $\mathbf{P}^{d}$ with a distinguished hyperplane (called $\infty$ ) that avoids $s$. The dual is an arrangement of hyperplanes $\mathbb{Q}$ in $\mathbf{P}^{d} ; \infty$ becomes a distinguished point lying in some region $R_{\infty}$. A separating hyperplane of $S$ becomes another point, in a region $R$ say; the induced partition of $S$ corresponds to the partition of $\mathbb{Q}$ given by $h \sim h^{\prime}$ iff $h$ and $h^{\prime}$ do not separate $R$ from $R_{\infty}$. Thus the non-Radon partitions of $S$ are in one-to-one correspondence with the regions of Q. Each region corresponds to an equivalence class of $S$-avoiding hyperplanes in $\mathbf{E}^{d}$ under the relation $k \sim k^{\prime}$ if $k$ can be moved continuously to $k^{\prime}$ without touching any point of $S$.

Let $L(S)$ be the lattice of affine subspaces generated by the points in $S$; it is the lattice of the affine dependence matroid of $S$. From Theorem B we conclude:

TheOrem 6.1 ([24, CoRollary 6.2], ALSo IN [6]). Let $S$ be a nonempty set of $n$ points in $\mathbf{E}^{d}$. The number of non-Radon partitions of $S$ is

$$
\frac{1}{2} \sum_{j} w_{j}^{+}(L(S))=\sum_{j} w_{j}^{\prime \prime+}(L(S)) .
$$

The number of Radon partitions is $2^{n-1}-\sum_{j} w_{j}^{\prime \prime+}(L(S))$.

Let us choose a distinguished "basepoint" $b \in S$ and regard the corresponding hyperplane $h(b)$ as the infinite; thus $Q$ becomes the projectivization of a Euclidean arrangement $\mathcal{E}=\mathscr{Q} \backslash\{h(b)\}$. A bounded region of $\mathcal{E}$ corresponds to an equivalence class of $S$-avoiding hyperplanes $k$ such that $k$ cannot be continuously shifted into a position containing $b$ without first passing through another point of $S$. Let us call 
such a $k$ barred from $b$. From Theorem $\mathrm{D}$ we have

THEOREM 6.2. Let $S \subseteq \mathbf{E}^{d}$ and let $b \in S$. Then the number of non-Radon partitions produced by hyperplanes barred from $b$ is equal to $\beta(L(S))$.

In order to interpret the $w_{j}^{\prime \prime+}(L(S))$ we need another definition. An affine subspace $t$ has general position with respect to $S$ if for every $x \in L(S), t$ and $x$ span the largest possible space and are not parallel.

THEOREM 6.3. Let $S$ span $\mathbf{E}^{d}$, let $b \in S$, and let $t_{-1}=\varnothing \subset t_{0} \subset t_{1} \subset \cdots \subset t_{d}=\mathbf{E}^{d}$ be a chain of affine subspaces in general position with respect to $S$. Then for $l=0,1, \ldots, d$, the number of non-Radon partitions of $S$ produced by hyperplanes $k \supseteq t_{l-1}$, but not by any hyperplane $k \supseteq t_{l}$, is equal to $w_{d-l}^{\prime \prime+}(L(S))$.

Proof. Let $g_{d-1-l}$ be the dual of $t_{l}$ in $\mathbf{P}^{d}$. A region $R$ of $Q$, the dual arrangement of $S$, meets $g_{d-1-l}$ if and only if $Q$ corresponds to a separating hyperplane $k \supseteq t_{l}$. Thus the theorem follows from Corollary 4.2.

Answering the question of Eckhoff [11, p. 170] on interpretation of the Whitney numbers, we have

COROLlary 6.1. The Whitney number $w_{d+1}^{+}(L(S))=\mu^{+}(L(S))$ equals the number of non-Radon partitions of $S$ not produced by any hyperplane through a fixed point $t_{0}$.

For $l=0,1, \ldots, d$, the Whitney number $w_{d-l}^{+}(L(S))$ equals the number of non-Radon partitions of $S$ produced by hyperplanes $k \supseteq t_{l-1}$, but not by any hyperplane $k \supseteq t_{l+1}$.

Proof. From Theorem 6.3 and (1.2).

The doubly indexed Whitney numbers are harder to interpret for Radon partitions. Consider an imperfectly separating hyperplane: one that meets $S$ in a set $S_{0}$ and separates the remainder into $S_{1}$ and $S_{2}$. Thus we have an imperfect non-Radon partition, into three parts of which $S_{0}$ is distinguished: we call it the middle part. The interpretation of Theorem B is

THEOREM 6.4. Let $S$ span $\mathbf{E}^{d}$ and let $-1 \leqslant i<d$ be arbitrary. The number of imperfect non-Radon partitions with $i$-dimensional middle is $\frac{1}{2} \sum_{j} w_{i+1, j}^{+}(S)$.

Proof. Again dualize to $\mathcal{Q}$ in $\mathbf{P}^{d}$; it has rank $d+1$. A hyperplane $k$ whose middle $S \cap k$ has dimension $i$ dualizes to a point in a cell of $\mathcal{Q}$ of dimension $d-(i+1)$; conversely each $d-(i+1)$-cell determines an imperfect non-Radon partition with middle dimension $i$. Thus the theorem.

Perhaps it would be more interesting to study "imperfect Radon partitions", that is tripartitions $\left\{S_{0} ; S_{1}, S_{2}\right\}$ of $S$ with $S_{0}$ distinguished such that $S \cap$ aff $S_{0}=S_{0}$ but no hyperplane containing $S_{0}$ separates $S_{1}$ from $S_{2}$. The number of these where $\operatorname{dim} S_{0}=i-1$ is

$$
N_{i}(S)=\sum_{T \in L(S)^{i}} 2^{n-1-|T|}-\frac{1}{2} \sum_{j=i}^{d+1} w_{i j}^{+}(L(S)) .
$$

(Here $T \in L(S)$ is understood to be a matroid-closed subset of $S$ rather than an affine subspace.) 
7. Acyclic orientations of graphs. A remarkable result of Stanley suggests that acyclic orientations of graphs can be treated through arrangements of hyperplanes.

Theorem 7.1 (Stanley [23]). Let $\Gamma$ be a graph with chromatic polynomial $\chi(\lambda)$. Then $\Gamma$ has exactly $|\chi(-1)|$ acyclic orientations.

We deduce Stanley's theorem from Theorem A after establishing the notation and the fundamental correspondences.

Say $\Gamma$ has the node set $N=\left\{p_{1}, p_{2}, \ldots, p_{n}\right\}$ and the edge set $E$; we write $e_{i j}$ for an (unoriented) edge with endpoints $p_{i}$ and $p_{j}$ and $(i, j)$ for $e_{i j}$ oriented from $p_{i}$ to $p_{j}$. (There may be more than one edge $e_{i j}$, but that will cause no difficulty.) To $e_{i j}$ corresponds a hyperplane $h_{i j}=\left\{x \in \mathbf{R}^{n}: x_{i}=x_{j}\right\}$. The graphic arrangement corresponding to $\Gamma$ is

$$
\mathcal{H}[\Gamma]=\left\{h_{i j}: e_{i j} \in E\right\} \text {. }
$$

Let $c=c(\Gamma)$ be the number of connected components of $\Gamma$. Then

$$
r(\mathcal{H}[\Gamma])=n-c
$$

and indeed

$$
\bigcap \mathscr{H}[\Gamma]=\left\{x \in \mathbf{R}^{n}: x_{i}=\text { constant in each component of } \Gamma\right\} ;
$$

in particular if $\Gamma$ is connected then $\cap \mathcal{H}[\Gamma]$ is the line $x_{1}=x_{2}=\cdots=x_{n}$. Further let $L(\Gamma)$ be the lattice of polygon-closed subsets of $E$, equivalently the lattice of partitions of $\Gamma$ (partitions $\pi$ of $N$ whose blocks induce connected subgraphs). This lattice is naturally isomorphic to $L(\mathcal{H}[\Gamma])$. The Whitney numbers $w_{i}$ appear in the chromatic polynomial:

$$
\chi(\lambda)=\sum_{j=0}^{n-c} w_{j}(L(\Gamma)) \lambda^{n-j} .
$$

For a set $S \subseteq E$ we count among the components of $S$ any isolated nodes. We assume $\Gamma$ has no loops; the case of loops is easily handled separately. A path is directed or coherent if, for each consecutive pair of arcs, one enters and the other leaves their common node. A coherent circle is a cycle.

LEMMA 7.1. There is a one-to-one correspondence between the acyclic orientations of $\Gamma$ and the regions of $\mathcal{H}[\Gamma]$, given by

$$
R(\alpha)=\left\{x \in \mathbf{R}^{n}: x_{i}<x_{j} \text { if } e_{i j} \text { is oriented }(i, j) \text { in } \alpha\right\}
$$

for each acyclic orientation $\alpha$, and inversely

$$
\alpha(R)=\left\{(i, j): e_{i j} \in E \text { and } x_{j}>x_{i} \text { if } x \in R\right\}
$$

for each region $R$.

Proof. Any $x \in \mathbf{R}^{n} \backslash \cup \mathcal{H}[\Gamma]$ defines an orientation $\alpha(x)$ by a rule like (7.3); clearly it is acyclic. Suppose $x$ moves continuously in $R$ : since at no time does $x$ cross a hyperplane $h_{i j} \in \mathcal{H}[\Gamma]$, there is no time at which any edge reverses direction. So $\alpha(R)$ is a well-defined acyclic orientation. 
Conversely given $\alpha$ we can show $R(\alpha) \neq \varnothing$, whence it follows from the previous paragraph that $R(\alpha)$ is a region. Writing $p_{i} \leqslant_{\alpha} p_{j}$ if $(i, j) \in \alpha$ (extended by transitivity), and extending this partial ordering of $N$ to a total ordering, say $p_{i_{1}}<\cdots<p_{i_{n}}$, we see that any $x$ whose coordinates are ordered $x_{i_{1}}<\cdots<x_{i_{n}}$ belongs to $R(\alpha)$.

Clearly $R(\alpha(R))=R$ and $\alpha(R(\alpha))=\alpha$.

Proof of Stanley's theorem. By the lemma $\Gamma$ has $f_{n}(\mathcal{F}[\Gamma])$ acyclic orientations; by $L(\mathcal{H}[\Gamma]) \cong L(\Gamma)$ and Theorem A this number is $\sum_{j} w_{j}^{+}(L(\Gamma))=|\chi(-1)|$.

LEMMA 7.2. There is a one-to-one correspondence between the acyclic orientations of all contractions $\Gamma / S$ where $S \in L(\Gamma)$ has $k$ components and the $k$-cells of $\mathcal{H}[\Gamma]$, given by

$$
C(\alpha, S)=\left\{x \in \mathbf{R}^{n}: x \in h_{i j} \text { if } e_{i j} \in S, x_{i}<x_{j} \text { if } e_{i j} \text { is oriented }(i, j) \text { in } \alpha\right\}
$$

for each acyclic orientation $\alpha$ of a contraction $\Gamma / S$, and inversely

$$
S(C)=\left\{e_{i j}: C \subseteq h_{i j}\right\}, \quad \alpha(C)=\left\{(i, j): x_{i}<x_{j} \text { if } x \in C\right\}
$$

for each cell $C$ of $\mathscr{H}[\Gamma]$.

COROLlaRY 7.1. The number of acyclic orientations of all contractions $\Gamma / S$ in which $S \in L(\Gamma)$ has $k$ components is equal to $\sum_{j} w_{n-k, j}^{+}(L(\Gamma))$.

The worth of the hyperplanar approach to acyclic orientations is that one can get other results by interpreting the geometry of selected regions. For instance from Theorem D by way of Theorem 3.4 we have Theorem 7.2. A source is a node with only outgoing arcs; a sink has only incoming arcs. We consider an isolated node to be neither a source nor a sink.

THEOREM 7.2. Let $e_{i j}$ be an arbitrary edge in $\Gamma$. The number of acyclic orientations of $\Gamma$ in which $p_{i}$ is the only source and $p_{j}$ is the only sink equals $\beta(L(\Gamma))$-regardless of the choice of edge $e_{i j}$.

PROof. If $\Gamma$ is disconnected, both quantities are 0 . So assume it is connected.

In Theorem 3.4 we set $\mathcal{H}=\mathcal{H}[\Gamma], h=h_{i j}$, and $h^{*}=\left\{x \in \mathbf{R}^{n}: x_{j}=x_{i}+1\right\}$. A region $R$ of $\mathcal{H}[\Gamma]$ meets $h^{*}$ iff $\alpha(R)$ orients $e_{i j}$ as $(i, j)$. It is relatively bounded in $\mathcal{H}_{h^{*}}$ iff the only way for any coordinate $x_{k}$ to become infinite while $x$ remains in $R$ is for $\sum x_{k}$ to become infinite.

Suppose $\alpha(R)$ has a source $p_{l} \neq p_{i}$. Then $x_{l}$ is unbounded below in $R$, so we can let $x_{l} \rightarrow-\infty$ and all other $x_{k} \rightarrow+\infty$ while holding $\sum x_{k}$ constant. Therefore $R$ is not relatively bounded in $\mathcal{H}_{h^{*}}$. A similar argument applies if there is a sink besides $p_{j}$.

On the other hand if $p_{i}$ is the only source and $p_{j}$ the only sink in $\alpha(R)$, then $x_{i}<x_{k}<x_{j}=x_{i}+1$ for every $x \in R$; therefore $R$ is relatively bounded in $\mathcal{H}_{h^{*}}$.

So by Theorem 3.4 we have the desired conclusion.

COROLlaRY 7.2. Let $e_{i j}$ be a fixed edge in $\Gamma$. The number of acyclic orientations of contractions $\Gamma / S$ in which $e_{i j} \notin S \in L(\Gamma)$ and $S$ has $k$ components, such that $p_{i}$ is the only source and $p_{j}$ is the only sink, equals $\sum_{j} w_{n-k, j}^{\prime \prime}\left(L\left(\Gamma, e_{i j}\right)\right)$.

PROOF. By Corollary 3.4 and arguing as in the preceding proof. 
THEOREM 7.3. Let $p_{i}$ be an arbitrary node of $\Gamma$. The number of acyclic orientations in which $p_{i}$ is the only source is equal to $w_{n-1}^{+}\left(L(\Gamma)\right.$ ) (which is $\mu^{+}(L(\Gamma)$ ) if $\Gamma$ is connected, 0 otherwise )-regardless of the choice of $p_{i}$.

Proof. We can assume $\Gamma$ is connected. Then the subspace $s=\left\{x: x_{i}=0\right\}$ sections $\mathscr{H}[\Gamma]$ faithfully and $g=\left\{x: \sum_{k \neq i} x_{k}=-1\right\}$ is a general hyperplane with respect to $\mathcal{E}=\mathscr{H}[\Gamma]_{s}$. By Theorem 3.1 then $g$ misses exactly $w_{n-1}^{+}(L(\Gamma))$ regions $R$ of $\mathcal{E}$.

If $p_{i}$ is the sole source in $\alpha(R)$, then all $x_{k}>x_{i}=0$ in $R$, so $R$ does not meet $g$. On the other hand if there is a source $p_{l} \neq p_{i}$, we can decrease $x_{l}$ at will until $\sum_{k \neq i} x_{k}<0$, then find a positive scalar multiple of $x$ in $g$, all the while keeping $x$ in $R$. Thus the regions not meeting $g$ are just those for which $\alpha(R)$ has the property of the theorem.

COROLlaRY 7.3. The number of acyclic orientations of all contractions $\Gamma / S$ in which $S \in L(\Gamma)$ has $k$ components, such that $p_{i}$ is the only source, equals $w_{n-k, n-1}^{+}(L(\Gamma))$.

Comparing Theorem 7.3 for $\Gamma$ to Theorem 7.2 for $\Gamma+p_{0}$ (that is, $\Gamma$ with an extra node $p_{0}$ adjacent to all other nodes) leads to the conclusion

$$
w_{n-1}^{+}(L(\Gamma))=\beta\left(L\left(\Gamma+p_{0}\right)\right) \text {. }
$$

For an acyclic orientation of $\Gamma$ with its only source at $p_{i}$ extends uniquely to an acyclic orientation of $\Gamma+p_{0}$ with its sole source and sink at $p_{i}$ and $p_{0}$. And an acyclic orientation of the latter type, restricted to $\Gamma$, has a source only at $p_{i}$.

One would expect there to be an interpretation of the other Whitney numbers $w_{j}^{+}(L(\Gamma))$ based on Corollary 3.2 , but we have not found a chain of subspaces whose geometry translates into graphically meaningful conditions.

What we do have is a purely graphical interpretation of the whole set of Möbius functions $\mu^{+}(0, \pi)$ for $\pi \in L(\Gamma)$. We need the nodes to be in a fixed order, say numerical order; and for $B \subseteq N$ let $\min B$ denote the first member of $B$. Recall the partial order $\leqslant_{\alpha}$ induced on $N$ by an acyclic orientation $\alpha$. For each $\alpha$ define a partition $\pi(\alpha)$ in this way: First set $\Gamma_{1}=\Gamma$; let $p_{i_{1}}$ be the last source in $\Gamma_{1}$ (in numerical subscript order) and $B_{1}$ the set of all nodes $p_{j}$ of $\Gamma_{1}$ reachable by an ascending path coherent in $\alpha$. Next let $\Gamma_{2}$ be the subgraph $\Gamma:\left[N \backslash B_{1}\right]$ induced on $N \backslash B_{1}$; repeat the construction on $\Gamma_{2}$ to get $p_{i_{2}}$ (the last source in $\Gamma_{2}$ ) and $B_{2}$. Let $\Gamma_{3}=\Gamma:\left[N \backslash\left(B_{1} \cup B_{2}\right)\right]$, and continue until all the nodes are used up: until $B_{1} \cup B_{2} \cup \cdots \cup B_{q}=N$. Then set

$$
\pi(\alpha)=\left\{B_{1}, B_{2}, \ldots, B_{q}\right\} .
$$

THEOREM 7.4. We have $\pi(\alpha) \in L(\Gamma)$, and each $\pi \in L(\Gamma)$ is the image of exactly $\mu^{+}(0, \pi)$ acyclic orientations.

Given $\alpha$ we have $p_{i_{m}}=\min B_{m}, p_{i_{m}}$ is the only source in $\Gamma: B_{m}$, and any edge with endpoints in different blocks $B_{1}$ and $B_{m}$ of $\pi(\alpha)$ is oriented against the order of minimal nodes (that is, from $B_{m}$ to $B_{l}$ if $i_{l}<i_{m}$, i.e., if $l>m$ ).

Conversely, given $\pi$ the following construction yields its complete inverse image: Orient each $\Gamma: B_{m}$ acyclically so that $\min B_{m}$ is the only source, and orient each edge between blocks against the order of minimal nodes. 
Proof. The construction of $\pi(\alpha)$ assures that each $\Gamma: B_{m}$ is connected, hence $\pi(\alpha) \in L(\Gamma)$. The other properties of $\pi(\alpha)$ are obvious. Thus when constructing orientations from $\pi(\alpha)$, one of those constructed is $\alpha$.

Conversely, if $\pi$ is given and $\alpha$ is constructed as described, then the source of the highest block $B_{1}$ is the highest source in $\alpha$, whence $\pi(\alpha)$ has $B_{1}$ for the highest block; stripping away blocks in succession and reasoning in the same way we see that $\pi(\alpha)=\pi$. We have therefore constructed the complete inverse image of $\pi$.

Its size follows from the observation that (by Theorem 7.3) there are $\mu^{+}\left(\Gamma: B_{m}\right)$ ways to orient each $\Gamma: B_{m}$ and, consequently,

$$
\mu^{+}(0, \pi)=\prod_{B \in \pi} \mu^{+}\left(\Gamma: B_{m}\right)
$$

ways to construct $\alpha$.

We can restate the definition of the blocks $B_{m}$. Let $\Gamma_{\alpha}$ be the subgraph of ascending arcs of $\alpha$ (ascending in the fixed ordering) and let $p_{i_{1}}, p_{i_{2}}, \ldots, p_{i_{4}}$ be the sources of $\Gamma_{\alpha}$ in descending order. Then

$$
B_{m}=\left\{p \in N: p \text { is reachable in } \Gamma_{\alpha} \text { from } p_{i_{m}} \text { but not from } p_{i_{1}}, \ldots, p_{i_{m-1}}\right\} .
$$

COROLlaRY 7.4. Given a fixed ordering of $N$, the number of acyclic orientations of $\Gamma$ in which $\Gamma_{\alpha}$ has exactly $q$ sources equals $w_{n-q}^{+}(L(\Gamma))$.

Taking $\Gamma=K_{n}$ we have a property of the retreating elements of a permutation $\left(a_{1}, a_{2}, \ldots, a_{n}\right)$ of $\{1,2, \ldots, n\}$, which are the $a_{i}$ such that $a_{1}, \ldots, a_{i-1}>a_{i}$. We call $a_{1}$ a retreating element.

Corollary 7.5 (Rényi [21]; cf. [8, Chapter VI, Exercise 10(3)]). The number of permutations of $\{1,2, \ldots, n\}$ having $q$ retreating elements equals $|s(n, q)|$, the unsigned Stirling number of the first kind.

Rényi stated this result in reverse, for "outstanding" elements (éléments saillants).

Lastly we offer an interpretation of certain sums of Mọbius functions that are not in general Whitney numbers. For $P, Q \subseteq N$, let

$$
E(P, Q)=\{e \in E: e \text { has one end in } P \text { and the other in } Q\} \text {. }
$$

Any bond (minimal cutset) of $\Gamma$ has the form $E\left(P, P^{c}\right)$, where $P^{c}=N \backslash P$. We say an orientation of $\Gamma$ directs a bond if it orients every bond edge in the same sense.

A null potential is a function $f: N \rightarrow \mathbf{R}$ such that $f(N)=0$, where

$$
f(P)=\sum_{p \in P} f(p) \text { for } P \subseteq N .
$$

We say such a function orients bonds by majority rule: if $E\left(P, P^{c}\right)$ is a bond with $f(P) \neq f\left(P^{c}\right)$, we orient it from the side whose value of $f$ is lower (hence negative) to the higher (positive) side. If $f(P)=f\left(P^{c}\right)$, we call $E\left(P, P^{c}\right)$ neutral and do not orient it. Clearly a bond is neutral if and only if $f(P)=0$. A partition $\pi \in L(\Gamma)$ is neutral if $f(B)=0$ for every $B \in \pi$ (equivalently if every bond $E\left(P, P^{c}\right)$ for which $\left\{P, P^{c}\right\} \geqslant \pi$ is neutral). The set $N(f)$ of neutral partitions is a nonempty modular filter in $L(\Gamma)$. 
THEOREM 7.5. Let $f$ be a null potential on $\Gamma$, not identically zero. The number of acyclic orientations of $\Gamma$ directing no bond against its f-orientation is equal to $\Sigma_{\pi \in N(f)} \mu^{+}(0, \pi)$.

Proof. We apply Theorem 3.5 to $\mathscr{H}[\Gamma]$ with $g$ the hyperplane

$$
g(x) \equiv \sum_{i} f\left(p_{i}\right) x_{i}=-1 .
$$

Since $\mathcal{H}[\Gamma]$ is central and its center is not in $g, M=\varnothing$. We must determine $N_{0}$. Consider the flat $t$ corresponding to $\pi \in L(\Gamma)$. The points $x \in t$ are characterized by $x_{i}=x_{B}$ for $p_{i} \in B$, so

$$
g(x)=\sum_{B \in \pi} f(B) x_{B} .
$$

Evidently $t$ meets $g$ if and only if some $f(B) \neq 0$. So the flats $t \in N_{0}$ are those corresponding to $\pi \in N(f)$.

Now pick $\alpha$ and suppose $E\left(P, P^{c}\right)$ is a bond directed by $\alpha$ from $P$ to $P^{c}$ and oriented oppositely by $f$; thus $f(P)>0$. Take $x \in R(\alpha)$. If we subtract $\lambda>0$ from each $x_{i}$ corresponding to $p_{i} \in P$, we do not remove $x$ from $R(\alpha)$, but we do decrease $g(x)$ by $\lambda f(P)>0$. Choosing $\lambda$ large enough makes $g(x)<0$. Then multiplying by a suitable scalar we obtain a point in $g \cap R(\alpha)$.

Inversely suppose $\alpha$ directs no bond against its $f$-orientation. Equivalently in the partial order $\leqslant_{\alpha}$ on $N$ we have $f(J) \geqslant 0$ for every order filter $J$. At this point we need a decomposition rule for a nondecreasing function $x: N \rightarrow \mathbf{R}$. Let $z_{1}>z_{2}>$ $\cdots>z_{m}$ be the values assumed by $x$. The set

$$
J_{k}=x^{-1}\left(\left\{z_{1}, \ldots, z_{k}\right\}\right), \text { for } k<m,
$$

is a filter. Letting $v_{k}=z_{k}-z_{k+1}>0$ and $1_{S}$ be the characteristic function of $S \subseteq N$, we have

$$
x=z_{m} 1_{N}+\sum_{k=1}^{m-1} v_{k} 1_{J_{k}} .
$$

Now since any $x \in R(\alpha)$ is an increasing function on $N$, calculating $g(x)$ by means of (7.5) yields

$$
g(x)=0+\sum_{k=1}^{n-1} v_{k} f\left(J_{k}\right) .
$$

But the right-hand side is nonnegative. So $g$ cannot meet $R(\alpha)$.

EXAmple 7.1. Digraph degrees. Let $(\Delta, \delta)$ be a digraph on $N$ and $f(p)$ be the net indegree of $p$ in $\delta$, that is

$$
f(p)=\operatorname{indeg}_{\delta}(p)-\operatorname{outdeg}_{\delta}(p) .
$$

Then the $f$-orientation of a bond $E_{\Gamma}\left(P, P^{c}\right)$ is the direction in which a majority of the arcs of $E_{\Delta}\left(P, P^{c}\right)$ go in $\delta$; it is neutral if there is no majority.

For instance let $\Delta$ be the star consisting of all edges at one vertex $p$, oriented outward by $\delta$. Then $N(f)=\left\{1_{\Gamma}\right\}$, and the acyclic orientations counted in Theorem 7.5 are those in which $p$ is the only source. So we have Theorem 7.3 as a corollary. 
Or suppose $\Delta$ is a smaller star, consisting of all edges between $p$ and $Q \subseteq N \backslash\{p\}$, directed outward by $\delta$. Then $N(f)$ is the set of partitions having a block containing $\{p\} \cup Q$, and the enumerated acyclic orientations are those in which $p$, but no member of $Q$, is a source.

EXAMPLE 7.2. Flows. A flow (or real voltage) on $\Gamma$ is a mapping $\varphi: E \rightarrow \mathbf{R}$, it being understood that $\varphi\left(e^{-1}\right)=-\varphi(e)$, where $e$ and $e^{-1}$ mean the same edge transversed in opposite directions. Its boundary $\partial \varphi$, defined by

$$
\partial \varphi(p)=\text { the net inflow to } p,
$$

is a null potential. Taking $f=\partial \varphi$ in Theorem 7.5, the $f$-orientation of a bond is in the "downhill" direction of flow. $N(f)$ consists of the partitions of which each block has no net inflow.

One could also take $\varphi$ to be a flow on a different graph $\Delta$ on the same node set $N$.

8. Totally cyclic orientations of graphs. An orientation of a graph $\Gamma$ is totally cyclic if every edge belongs to a (directed) cycle. For a connected graph this means just that the orientation is strongly connected. The numbers appropriate for counting totally cyclic orientations are the Whitney numbers of the lattice $L^{\perp}(\Gamma)$ of the cographic matroid, whose closed sets are the complements of the isthmus-free subsets of $E$. We wish to treat totally cyclic orientations geometrically; for that we need the cographic arrangement of hyperplanes associated with $\Gamma$.

We assume in this section that $\Gamma$ has no isthmi. By complicating our definitions slightly we could allow isthmi, but we prefer to avoid the extra complexity.

We start the construction in $\mathbf{R}^{E}$, whose coordinates are $x(e)$ for $e \in E$ with the convention $x\left(e^{-1}\right)=-x(e)$ as in Example 7.2. Let

$$
\partial x(P)=\text { the net inflow to } P=\sum_{p \in P} \partial x(p) \text { for } P \subseteq N .
$$

The cycle space of $\Gamma$ is $u=\left\{x \in \mathbf{R}^{E}: \partial x=0\right\}$. Writing $\mathcal{Q}$ for the arrangement of coordinate hyperplanes in $\mathbf{R}^{E}$, we define the cographic arrangement of hyperplanes of $\Gamma$ to be the induced arrangement, $\mathcal{H}^{\perp}[\Gamma]=\mathcal{Q}_{u}$, and we write $h(e)$ for the hyperplane corresponding to $e$. Notice that this arrangement is central. A fact that is in essence well known is that

$$
L\left(\mathfrak{H}^{\perp}[\Gamma]\right)=L^{\perp}(\Gamma) .
$$

Any region $R$ of $\mathcal{H}^{\perp}[\Gamma]$ (or for that matter of $\mathcal{Q}$ ) determines an orientation $\tau(R)$ of $\Gamma$ by the rule: pick $x \in R$ and choose the direction of $e$ that makes $x(e)>0$.

LEMMA 8.1. The mapping $R \rightarrow \tau(R)$ is a one-to-one correspondence between the regions of $\mathcal{H}^{\perp}[\Gamma]$ and the totally cyclic orientations of $\Gamma$.

Consider a region $R$. (We assume $\Gamma$ has no isthmi.) We can orient $\Gamma$ so all $x(e)$ are positive in $R$. If $\tau(R)$ were not totally cyclic, there would be a bond $E\left(P, P^{c}\right)$ directed by $\tau(R)$ from $P$ to $P^{c}$. Since $x \in u$, we would have $\partial x(P)=0$. But all $x(e)>0$. So there is a contradiction.

Conversely let $\tau$ be a totally cyclic orientation. Assign a positive number $a(C)$ to each cycle and let $x(e)=\Sigma a(C)$, summed over all cycles containing $e$. Since $\partial x=0$, we have found a region $R$, namely that containing $x$, for which $\tau(R)=\tau$. 
Now from Theorem A we have

THEOREM 8.1. The number of totally cyclic orientations of an isthmus-free graph $\Gamma$ is equal to $\sum_{i} w_{i}^{+}\left(L^{\perp}(\Gamma)\right)$.

The nullity of $S \subseteq E$ is

$$
\operatorname{nul}(S)=\# S-c(N, S) .
$$

We have $\operatorname{dim} u=\operatorname{nul}(\Gamma)$.

LEMMA 8.2. There is a one-to-one correspondence between the pairs $\left(S, \tau_{S}\right)$, where $S$ is an isthmus-free edge set in $\Gamma$ of nullity $k$ and $\tau_{S}$ is a totally cyclic orientation of $S$, and the $k$-cells of $\mathcal{H}^{\perp}[\Gamma]$, given by

$$
C\left(S, \tau_{S}\right)=\left\{x \in u: x(e)=0 \text { for } e \notin S, x(e)>0 \text { for } e \in S \text { as oriented by } \tau_{S}\right\} \text {. }
$$

The lemma follows from (8.1) and Lemma 8.1.

COROLlARY 8.1. The number of totally cyclic orientations of all isthmus-free edge sets in $\Gamma$ of nullity nul $(\Gamma)-i$ is equal to $\sum_{j} w_{i j}^{+}\left(L^{\perp}(\Gamma)\right)$.

A circle $C \subseteq E$ determines a vector $x_{C} \in \mathbf{R}^{E}$ in the following way: Choose a direction around $C$; then (for purposes of definition) orient each $e \in C$ to agree with that direction and let $x_{C}(e)=1$ if $e \in C, x_{C}(e)=0$ if $e \notin C$. We see that $x_{C} \in u$. Note that choosing the opposite direction for $C$ would negate $x_{C}$.

If $\tau$ is an orientation of $\Gamma$, we write $\tau_{e}$ for the orientation obtained by reversing $e$.

LEMMA 8.3. The boundary hyperplanes of a region $R$ of $\mathcal{H}^{\perp}[\Gamma]$ are the $h(e)$ for which $\tau(R)_{e}$ is totally cyclic.

THEOREM 8.2. Let $e \in E(\Gamma)$. The number of totally cyclic orientations of $\Gamma$ in which $e$ has a fixed orientation and every cycle passes through e is equal to $\beta\left(L^{\perp}(\Gamma)\right)$.

FIrst Proof (by duality). Assume $\Gamma$ has more than one edge; the other case is easy. The orientations are in bijection via the map $\tau \rightarrow \tau_{e}$ with the acyclic orientations in which $e$ has the opposite fixed orientation and its endpoints are the only source and sink. The latter number is $\beta(L(\Gamma))$ by Theorem 7.2, which equals $\beta\left(L^{\perp}(\Gamma)\right)$ by Crapo's duality theorem [10, Theorem IV].

SECOND Proof (by geometry). Taking $e$ in its fixed orientation, we set $h=h(e)$ and $h^{*}=\{x \in u: x(e)=1\}$ in Theorem 3.4. Then $\beta\left(L^{\perp}(\Gamma)\right)$ counts the regions $R$ such that $\tau(R)$ gives $e$ the fixed direction and $R \cap h^{*}$ is bounded. If $x \in R \cap h^{*}$ and $\tau(R)$ has a cycle $C$ not containing $e$, then $x+\lambda x_{C} \in R \cap h^{*}$ for any $\lambda \geqslant 0$, so $R \cap h^{*}$ is unbounded.

Suppose, conversely, that $R \cap h^{*}$ is unbounded and $\{x+\lambda y: \lambda \geqslant 0\}$ is a ray in $R \cap h^{*}$. Thus $y \in u$ and $y(e)=0$. Since $y \neq 0$, there must be a cycle of $\tau(R)$ whose edges have nonzero values of $y$. But no such cycle contains $e$. So $\tau(R)$ does not fit the requirements of the theorem.

To interpret $\mu\left(L^{\perp}(\Gamma)\right)$ we need an auxiliary item: a certain kind of orientation of the circles of $\Gamma$. Let $g_{0}$ be a hyperplane through the origin of $\mathbf{R}^{E}$, general with respect to the coordinate arrangement $Q$. Then $g=g_{0} \cap u$ is general through the origin with 
respect to $\mathcal{H}^{\perp}[\Gamma]$. Choose a positive side of $g$ and let $\gamma$ orient each circle $C$ in such a way that $x_{C}$ is on the positive side. (Reminder: $\gamma$ does not orient edges.) We call such a way of orienting the circles linear. An intrinsic characterization is

PROPOSITION 8.1. Let $\gamma$ be a circle orientation and $x_{C}$, for each circle $C$, the vector corresponding to the orientation by $\gamma$. For $\gamma$ to be linear it is necessary and sufficient that the only nonnegative linear combination $\Sigma b_{C} x_{C}=0$ is that with all $b_{C}=0$.

Proof. One can see that $\gamma$ is linear if and only if there is a hyperplane $g^{*}$ separating all $x_{C}$ from 0 . The latter exists if and only if 0 is not a positive combination of any $x_{C}$.

Here is an open question. Acyclic and totally cyclic orientations are each characterized by a simple excluded configuration (cycles, and sources or sinks, respectively). To what extent can linear circle orientations be characterized in the same way? One forbidden configuration is a theta graph in which every two circles are oriented oppositely on their common path. We believe this one exclusion is too weak for a characterization, although we have no confirming example. Is there a short sufficient list of forbidden configurations?

The support of a vector $x \in \mathbf{R}^{E}$ is

$$
\operatorname{supp} x=\{e: x(e) \neq 0\} .
$$

Let $\tau(x)$ be the orientation of supp $x$ that makes all $x(e) \geqslant 0$.

LEMMA 8.4. Each $x \in u$ is a positive combination of vectors $x_{C}$ belonging to cycles in $\tau(x)$.

Proof. We induct on \# supp $x$. By Corollary $8.1 \tau(x)$ is a totally cyclic orientation of $\operatorname{supp} x$. Let $C$ be a cycle passing through an edge of minimal weight $a=|x(e)| \neq 0$. Then $\tau\left(x-a x_{C}\right)$ agrees with $\tau(x)$ where both are defined, and $x-a x_{C}$ is a positive combination of cycles. So we have the lemma.

As a byproduct we have the well-known fact that $u$ is the linear span $u=\left\langle x_{C}: C\right.$ is a circle of $\Gamma\rangle$. Another conclusion is

Lemma 8.5. Let $R$ be a region of $\mathcal{K}^{-\perp}[\Gamma]$ and $\bar{R}$ its closure. Let $x \in u$. Then $x \in \vec{R}$ if and only if $x$ is a nonnegative combination of vectors $x_{C}$ belonging to cycles of $\tau(R)$, and $x \in R$ if and only if also the cycles with positive coefficient $b_{C}$ cover $E$.

Proof. The criterion for $x \in \bar{R}$ is immediate from Lemma 8.4. That for $x \in \bar{R}$ follows by taking into account the support of $x$.

A corollary is that the edges of $R$ are the rays $\left\langle x_{C}\right\rangle^{+}=\left\{\lambda x_{C}: \lambda>0\right\}$ for which $C$ is a cycle in $\tau(R)$.

THEOREM 8.3. Let $\gamma$ be a linear circle orientation of $\Gamma$. The number of totally cyclic orientations of $\Gamma$ whose every cycle is oriented as prescribed by $\gamma$ equals $\mu^{+}\left(L^{\perp}(\Gamma)\right)$.

Proof. Say $\gamma$ corresponds to the half space $a \cdot x>0$, Let $g$ be the hyperplane $a \cdot x=-1$, general with respect to $\mathcal{H}^{\perp}[\Gamma]$. According to Theorem $3.1, g$ misses exactly $\mu^{+}\left(L^{\perp}(\Gamma)\right)$ regions. 
By Lemma 8.5,g meets a closed region $\bar{R}$ if and only if there is a point $x=\Sigma b_{C} x_{C}$ (summed over cycles of $\tau(R)$ ), where the $x_{C}$ are oriented to agree with $\tau(R)$ and the $b_{C}$ are nonnegative, for which

$$
-1=a \cdot x=\sum b_{C}\left(a \cdot x_{C}\right) .
$$

This equation has a solution precisely when some $a \cdot x_{C}<0$, in other words $C$ is oriented oppositely by $\tau(R)$ and $\gamma$. Since $g$ is general, it meets $R$ under the same conditions. The theorem follows.

COROLlary 8.2. Let $\Gamma$ be a directed graph. Order the edges of $\Gamma$ in a fixed way. The number of totally cyclic reorientations $\tau$ of $\Gamma$, such that in each cycle of $\tau$ the lowest edge is not reoriented, is equal to $\mu^{+}\left(L^{\perp}(\Gamma)\right)$.

PRoof. Let the edge ordering be $e_{1}<e_{2}<\cdots<e_{|E|}$. The appropriate circle orientation $\gamma$ is linear: it corresponds to choosing the coefficients $a_{1}, a_{2}, \ldots, a_{\mid t]}$ of the general hyperplane $g$ so that $a_{1} \gg a_{2} \gg \cdots \gg a_{|E|}>0$.

So far we have not found how to extend Theorem 8.3 to interpret the Whitney numbers of Möbius function values of $L^{\perp}(\Gamma)$. This is the main outstanding problem in our approach to totally cyclic orientations.

A plane graph has a natural circle orientation $\gamma$, in which every cycle is oriented counterclockwise. That $\gamma$ is linear follows easily from Proposition 8.1. Now Theorem 8.3 gives us

Corollary 8.3. Let $\Gamma$ be a graph embedded in the plane. The number of totally cyclic orientations of $\Gamma$ in which there is no clockwise cycle equals $\mu^{+}\left(L^{\perp}(\Gamma)\right)$ if $\Gamma$ is isthmus free, 0 otherwise.

The circle orientation that yields this corollary depends first of all on embedding $\Gamma$ in the sphere, then on choosing a face $F$ in which to puncture the sphere. This $F$ defines $\gamma$ and becomes the unbounded face of the plane embedding. However we could determine $\gamma$ using one face and puncture a different one.

COROLlaRY 8.4. Let $\Gamma$ be a graph embedded in the plane and $F$ a bounded face of the embedding. Consider the totally cyclic orientations of $\Gamma$ in which each cycle that surrounds $F$ is directed clockwise while each other cycle is counterclockwise. The number of such orientations is $\mu^{+}\left(L^{\perp}(\Gamma)\right)$ if $\Gamma$ is isthmus free, 0 otherwise.

Proof. We regard $\Gamma$ as embedded in the Riemann sphere. Let $F_{0}$ be the face that becomes unbounded in the plane. To define $\gamma$ we orient each circle of $\Gamma$ so that, viewed in the sphere, it wraps clockwise around $F$. The corollary now follows from Proposition 8.1 and Theorem 8.3.

We might ask whether Theorem 7.3 on acyclic orientations would yield interesting results for plane graphs. It does indeed do so if one applies it to the dual graph. But one gets the same results more simply by combining Corollaries 8.3 and 8.4 with the following structural propositions.

Proposition 8.2. Let $\Gamma$ be as in Corollary 8.3. Consider a totally cyclic orientation of $\Gamma$ in which no boundary of a bounded face is directed clockwise. Such an orientation has no clockwise cycles. Moreover it directs the outer boundary of $\Gamma$ counterclockwise. 
Proposition 8.3. Let $\Gamma$ and $F$ be as in Corollary 8.4. Consider a totally cyclic orientation of $\Gamma$ in which no boundary of a bounded face other than $F$ is directed clockwise and the outer boundary is not directed counterclockwise. In each such orientation a cycle is directed clockwise if it surrounds $F$, counterclockwise if it does not. Moreover the boundary of $F$ is directed clockwise.

Proofs. We work through the dual graph $\Gamma^{*}$ by means of a standard correspondence between orientations $\tau$ of $\Gamma$ and $\tau^{*}$ of $\Gamma^{*}$. Given $\tau$, if $e^{*}$ is the edge of $\Gamma^{*}$ corresponding to $e \in E(\Gamma)$, then $\tau^{*}$ orients $e^{*}$ so it crosses $e$ from left to right as one looks along $e$ in the forward direction. (Notice that $\left(\tau^{*}\right)^{*}=\tau^{-1}$, the reverse of $\tau$.) It is well known that $\tau^{*}$ is acyclic if (and only if) $\tau$ is totally cyclic. For completeness we give the easy proof. Suppose $\tau^{*}$ has a cycle $C^{*}$. Then the dual edge set $C \subseteq E(\Gamma)$ constitutes a cut set separating (say) $X$ from $Y=N(\Gamma) \backslash X$ such that every edge in $C$ is directed from $X$ to $Y$. Clearly no $e \in C$ can belong to a cycle in $\tau$. Hence $\tau$ is not totally cyclic.

Let $\tau$ be one of the totally cyclic orientations considered in Propositions 8.2 and 8.3. A node $p^{*}$ of $\Gamma^{*}$ is a source if and only if the boundary of its corresponding face $F$ of $\Gamma$ is directed clockwise (for $F \neq$ the unbounded face $F_{0}$ ) or counterclockwise (for $F=F_{0}$ ). Since $\tau^{*}$ is acyclic it must have a source $p^{*}$, whose corresponding face can only be $F_{0}$ in Proposition 8.2, $F$ in Proposition 8.3. Thus the outer boundary, or the boundary of $F$, are as described.

Suppose $\tau$ has a clockwise cycle $C$. Then the dual edge set $C^{*}$ is directed outward from the nodes of $\Gamma^{*}$ lying inside $C$. Since $\tau^{*}$ is acyclic, one of these nodes $p^{*}$ must be a source. Since the corresponding face is bounded, we must be in the case of Proposition 8.3 and the face is $F$. Hence $C$ surrounds $F$. On the other hand suppose $C$ is a counterclockwise cycle of $\tau$. Then $C^{*}$ is directed into the set of nodes within $C$, so there is a source $p^{*}$ of $\tau^{*}$ outside $C$. In Proposition $8.3, p^{*}$ must correspond to $F$; hence $C$ does not surround $F$.

9. Acyclic orientations of signed graphs. A signed graph $\Sigma$, consisting of a graph $\Gamma$ and a sign labelling $\sigma: E \rightarrow\{ \pm\}$, has a matroid $G(\Sigma)$ and hence a geometric lattice of flats $L(\Sigma)$, whose Whitney numbers $w_{i j}^{+}(L(\Sigma)$ ) count the acyclic orientations of $\Sigma$ just as for ordinary graphs. Because ordinary graphs are essentially the same as all-positive signed graphs, one can expect to find signed-graphic generalizations of the results of the two previous sections. But the generalizations are not always straightforward extensions, and they seem to be consistently harder to prove. We have only found the analog of Stanley's theorem and interpretations of the Möbius and beta invariants.

To describe the matroid and lattice of $\Sigma$ we need to define balance. ${ }^{2}$ A circle is balanced if its sign product is +; an edge set is balanced if every circle in it is balanced. A circuit in $G(\Sigma)$ is either a balanced circle or a pair of unbalanced circles and a simple connecting path meeting each circle only at an endpoint. (If the circles

\footnotetext{
${ }^{2}$ The material on the matroid and lattice is from [28], on orientation from [29]. Here we omit half edges and free loops; for our purposes they are equivalent, respectively, to negative and positive loops (cf. $[28,29])$.
} 
meet, they can do so only at one node; then the path has length 0 .) Let $b(S)$ be the number of balanced components of $S \subseteq E$, counting isolated nodes, and $n=\# N$ as usual. The rank function in $G(\Sigma)$ is

$$
\operatorname{rk}(S)=n-b(S) \text {. }
$$

An edge set $S$ is a flat if its unbalanced components form an induced subgraph of $\Sigma$ and no balanced circle has all but one edge in $S$. To make the empty set closed (and our results correct as stated here), we assume $\Sigma$ has no balanced loops.

One orients $\Sigma$ by putting two arrows on each edge $e$, pointing in the same sense if $\sigma(e)=+$, the opposite sense otherwise. We describe an orientation $v$ by attaching a sign to each end of $e: v(e, p)=+$ if $e$ enters the endpoint $p,-$ otherwise. A cycle is a (matroid) circuit with no terminus (source or sink).

The signed-graphic arrangement $\mathcal{H}[\Sigma]$ lies in $\mathbf{R}^{n}$. The hyperplane corresponding to an edge $e_{i j}$ is $h\left(e_{i j}\right): x_{j}=\sigma\left(e_{i j}\right) x_{i}$. Then $L(\mathcal{H}[\Sigma])$ is naturally isomorphic to $L(\Sigma)$, as shown in [28, Theorem 8B.1]. The generalization to signed graphs of Lemma 7.1 is easy to state but the proof is long, occupying the bulk of [29].

LEMMA 9.1 [29, THEOREM 4.2]. There is a one-to-one correspondence between the acyclic orientations of $\Sigma$ and the regions of $\mathcal{H}[\Sigma]$, given by

$$
R(\alpha)=\left\{x \in \mathbf{R}^{n}: \alpha\left(e_{i j}, p_{i}\right) x_{i}+\alpha\left(e_{i j}, p_{j}\right) x_{j}>0 \text { for all } e_{i j} \in E\right\}
$$

for each acyclic orientation $\alpha$, and inversely $\alpha(R)$ given by

$$
\alpha(R)\left(e_{i j}, p_{i}\right)=\operatorname{sgn}\left[x_{i}-\sigma\left(e_{i j}\right) x_{j}\right], \quad \text { if } x \in R,
$$

for each region $R$.

TheOrem 9.1 [30, Corollary 4.1]. The number of acyclic orientations of a signed graph $\Sigma$ equals $\sum_{j} w_{j}^{+}(L(\Sigma))$.

For a geometric proof, apply Theorem A to Lemma 9.1. A graphical proof appears in [30].

For our interpretations of $\mu$ and $\beta$ we need another definition. A half-cycle at $p \in N$ is an unbalanced circle with a simple path of length $\geqslant 0$ attached to it ane end, oriented so that $p$ is the only terminus.

THEOREM 9.2. In a signed graph $\Sigma$, choose an edge $e$ and give it a fixed orientation. The number of acyclic orientations of $\Sigma$ giving $e$ the fixed orientation, having no termini outside the endpoints of $e$, and (if $\Sigma$ is unbalanced) having a half-cycle at each endpoint $p$ of $e$ with the same direction at $p$ as $e$ has, is equal to $\beta(L(\Sigma))$.

Proof. We rely on machinery from [29], to which we refer for the definitions.

If $\Sigma$ is balanced, Theorem 7.2 applies after switching $\Sigma$ so it is all positive. If $\Sigma$ is disconnected (neglecting isolated nodes), then $\beta=0$ and there are no suitable orientations. So we may assume $\Sigma$ has no balanced components.

Let $p_{i}$ and $p_{j}$ be the endpoints of $e$. (Possibly $p_{i}=p_{j}$.) By suitable switching we can assume $e$ is negative and extroverted. Then $h(e)$ is the hyperplane $x_{i}+x_{j}=0$; to apply Theorem 3.4 we take

$$
h^{*}: x_{i}+x_{j}=1
$$


Let $\alpha$ be an acyclic orientation orienting $e$ correctly. When is $R(\alpha) \cap h^{*}$ bounded? If $\alpha$ has a source (or sink) $p_{k}$, not an endpoint of $e$, then $x_{k}$ is not bounded below (or above) for $x \in R(\alpha)$, so $R(\alpha) \cap h^{*}$ is unbounded. Thus we suppose from now on that $e$ has no termini other than $p_{i}$ and $p_{j}$.

Now $\tilde{\Sigma}$ is oriented by $\tilde{\alpha}$, the lift of $\alpha$. To have a lower bound on a coordinate $x_{k}^{\varepsilon}$, there must be an arc entering $\varepsilon p_{k}$. Tracing back in a directed path as far as possible, we arrive at a source, which must be $-p_{i}$ or $-p_{j}$. So every $x_{k}^{\varepsilon}$ for $k \neq i, j$ is bounded below (and similarly above) by one of $x_{i}^{+}, x_{i}^{-}, x_{j}^{+}$, and $x_{j}^{-}$. Therefore for $R(\alpha) \cap h^{*}$ to be bounded, it is necessary and sufficient that these four values be bounded in $\tilde{R}(\tilde{\alpha}) \cap s \cap h^{*}$. At this point the case $p_{i}=p_{j}$ becomes trivial, so we assume $p_{i} \neq p_{j}$ from now on.

We have three equations in $\tilde{R}(\tilde{\alpha}) \cap s \cap h^{*}$ :

$$
x_{i}^{+}+x_{j}^{+}=1, \quad x_{i}^{-}=-x_{i}^{+}, \quad x_{j}^{-}=-x_{j}^{+} .
$$

Other relations among these variables can only appear from directed paths among $\pm p_{i}$ and $\pm p_{j}$. The possibilities are the following:

(1) A directed path from $-p_{i}$ to $+p_{j}$ (or $-p_{j}$ to $+p_{i}$ ) gives no new constraints.

(2) A directed path from $-p_{i}$ to $+p_{i}$ (or $-p_{j}$ to $+p_{j}$ ) forces $x_{i}^{+}>0$, hence $x_{j}^{+}<1$ (or $x_{j}^{+}>0$ and $x_{i}^{+}<1$ ).

(3) A directed path from $+p_{i}$ to $+p_{j}$ extends, using $\tilde{e}$, to one from $-p_{j}$ to $+p_{j}$, so it need not be considered separately.

We conclude that $\tilde{R}(\tilde{\alpha}) \cap s \cap h^{*}=R(\alpha) \cap h^{*}$ is bounded if and only if both possibilities in (2) occur. But existence of a directed path in $\tilde{\Sigma}$ from $-p_{i}$ to $+p_{i}$ is equivalent to existence of a half-cycle into $p_{i}$ in $\Sigma$. Hence the theorem.

Suppose the nodes are ordered. We call $p$ an upward node if there is no directed path entering $p$ with its other end at a higher node.

THEOREM 9.3. Let $\Sigma$ be a signed graph with no balanced components whose nodes are linearly ordered. The number of acyclic orientations such that every upward node has an entering half-cycle is equal to $\mu^{+}(L(\Sigma))$.

Proof. Say the nodes are in subscript order $p_{1}, p_{2}, \ldots, p_{n}$, and $a_{1}, a_{2}, \ldots, a_{n}$ are real numbers satisfying $0<a_{1} \ll a_{2} \ll \cdots \ll a_{n}$. We apply Theorem 3.1 with $g$ given by the equation $\sum_{i} a_{i} x_{i}=-1$. That is, we want to characterize the acyclic orientations $\alpha$ such that no $x \in R(\alpha)$ has $\sum_{i} a_{i} x_{i}<0$.

First we observe that an acyclic orientation that misses $g$ can have no source. For if, say, $x \in R(\alpha)$ and $p_{i}$ were a source, then $x_{i}$ could be decreased to make $\sum_{i} a_{i} x_{i}<0$ while keeping $x \in R(\alpha)$. Henceforth we assume $\alpha$ has no source.

Suppose $\alpha$ has an upward node $p_{i}$ with no entering half-cycle. We show that $\Sigma_{i} a_{i} x_{i}$ can be made negative starting from an $x \in R(\alpha)$ by reducing $x_{i}$ and altering certain other coordinates of $x$ to keep the vector in $R(\alpha)$. Consider the sets $A_{\varepsilon}$ of nodes $p_{k}$ for which there is a coherent path $P_{\varepsilon}\left(p_{k}\right)$ with sign product $\varepsilon$, entering $p_{i}$, and having $p_{k}$ for its other endpoint. (For instance $p_{i} \in A_{+}$.) We have $A_{-} \neq \varnothing$ because $p_{i}$ cannot be a source. In addition, $A_{+} \cap A_{-}=\varnothing$. To prove this we first show that any path $P_{\varepsilon}\left(p_{k}\right)$ is simple. If not, let $p_{j}$ be the first repeated node in it and let $P^{\prime}$ be the 
initial segment of $P_{\varepsilon}\left(p_{k}\right)$ up to the first repetition of $p_{j}$. Then $P^{\prime}$ is a circle, coherent except perhaps at $p_{j}$, with a tail of length $\geqslant 0$ extending to $p_{i}$. If the circle is balanced, it is a cycle; if unbalanced, we have a half-cycle entering $p_{i}$; but neither is permitted. So $P_{\varepsilon}\left(p_{k}\right)$ must be simple. Now if $A_{+} \cap A_{-} \neq \varnothing$, there are paths $P_{+}\left(p_{k}\right)$ and $P_{-}\left(p_{k}\right)$. Let $p_{j}$ be the first point at which they diverge and $p_{l}$ the first following point of $P_{+}\left(p_{k}\right)$ at which it meets $P_{-}\left(p_{k}\right)$. Then the segments of $P_{+}\left(p_{k}\right)$ and $P_{-}\left(p_{k}\right)$ from $p_{j}$ to $p_{l}$ form a circle $Q$. If it is balanced, we can replace the segment of $P_{-}\left(p_{k}\right)$ from $p_{j}$ to $p_{l}$ by the segment of $P_{+}\left(p_{k}\right)$ and repeat the argument. Eventually we must find an unbalanced circle $Q$, which together with the common initial segment up to $p_{j}$ forms a half-cycle entering $P_{i}$. But this is a contradiction. So $A_{+}$ and $A_{-}$are disjoint.

Now we take $x \in R(\alpha)$ and modify it to $z$ by setting

$$
z_{j}= \begin{cases}x_{j}-\lambda & \text { if } p_{j} \in A_{+}, \\ x_{j}+\lambda & \text { if } p_{j} \in A_{-}, \\ x_{j} & \text { if } p_{j} \notin A_{+} \cup A_{-},\end{cases}
$$

where $\lambda \geqslant 0$. Let $\varepsilon_{j}=+1,-1,0$ in these respective cases. Consider a constraint $\delta z_{j}+\delta^{\prime} z_{k}>0$ imposed by an edge $e_{j k}$. It is satisfied by $x$; therefore it is satisfied by $z$ unless $\delta \varepsilon_{j}+\delta^{\prime} \varepsilon_{k}>0$. If the latter holds, then (say) $\delta=\varepsilon_{j}$, which implies that the coherent path entering $p_{i}$ with endpoint $p_{j}$ can be extended to $p_{k}$. Hence $\varepsilon_{k} \neq 0$, indeed $\varepsilon_{k}=\sigma\left(e_{j k}\right) \varepsilon_{j}=-\delta \delta^{\prime} \varepsilon_{j}$. But that says $\delta \varepsilon_{j}+\delta^{\prime} \varepsilon_{k}=0$, so $z$ satisfies the edge constraint. We conclude $z \in R(\alpha)$.

Since $p_{i}$ is an upward node, $a_{i} \gg a_{j}$ for all $p_{j} \in A_{+} \cup A_{-}$besides $p_{i}$. So if we let $\lambda \rightarrow \infty, \sum_{i} a_{i} z_{i}$ is dominated by $-\lambda a_{i} \rightarrow-\infty$. Taking $\lambda$ sufficiently large, we get $z \in R(\alpha)$ for which $\sum_{i} a_{i} z_{i}<0$. Thus if $\alpha$ has an upward node without an entering half-cycle, it does not have the geometry we want.

The remaining task is to prove that, if $\alpha$ does have a half-cycle entering each upward node, then $\Sigma_{i} a_{i} x_{i}>0$ for all $x \in R(\alpha)$. If there is a directed path in $\tilde{\Sigma}$ from $-p_{i}$ to $+p_{i}$, then $x_{i}=x_{i}^{+}>0$ for all $x \in R(\alpha)$. This is the case if $p_{i}$ has an entering half-cycle. If on the other hand $p_{j}$ has no such half-cycle, it is not an upward node. So there is a higher upward node $p_{f(j)}$ at the end of a path entering $p_{j}$. Now consider a node $p_{i}$ with an entering half-cycle. For all $j \in f^{-1}(i)$ we have $a_{j} \ll a_{i}$. We also have $x_{i}>0$ and $x_{j}> \pm x_{i}$ (the sign depending on the path from $p_{i}$ entering $p_{j}$ ) for all $x \in R(\alpha)$. So

$$
a_{i} x_{i}+\sum_{j \in f^{-1}(i)} a_{j} x_{j}>\left[a_{i}+\sum_{j \in f^{-1}(i)}\left( \pm a_{j}\right)\right] x_{i}
$$

Because $a_{i} \gg a_{j}$ for all $j<i$, the bracketed expression is positive. We conclude that $\sum_{i} a_{i} x_{i}>0$ for $x \in R(\alpha)$.

NOTE. The basic results here date from 1975 and were announced in [13] and [27]. For the delay in preparing this article the authors apologize to their readers and to each other. 


\section{REFERENCES}

1. G. L. Alexanderson and John E. Wetzel, Dissections of a plane oval, Amer. Math. Monthly 84 (1977), 442-449. MR 58 23976; Zbl. 375.50009.

2. __ Arrangements of planes in space, Discrete Math. 34 (1981), 219-240. MR 82i: 51028: Zbl. 477.05021.

3. Gerald Berman, The dichromate and orientations of a graph, Canad. J. Math. 29 (1977), 947-956. MR 57 \#9591: Zbl. 378.05031.

4. R. G. Bland and M. Las Vergnas, Orientability of matroids, J. Combin. Theory Ser. B 24 (1978), 94-123. MR 58 \# 5294: Zbl. 374.05016.

5. Thomas Brylawski, A decomposition for combinatorial geometries, Trans. Amer. Math. Soc. 171 (1972), 235-282. MR 46 \#869; Zbl. 224.05007 (248.05019).

6. A A combinatorial perspective on the Radon convexity theorem, Geometriae Dedicata 5 (1976), 459-466. MR 55 \# 13340; Zbl. 361.52002.

7. Thomas H. Brylawski and Dean Lucas, Uniquely representable combinatorial geometries. Colloq Internat. Teorie Combinatorie (Roma, 1973), Tomo I, Atti dei Convegni Lincei, No. 17, Accad. Naz. Lincei, Rome, 1976, pp. 83-104. Zbl. 392.51007.

8. Louis Comtet. Advanced combinatorics, Reidel, Dordrecht, 1974. MR 57 124: Zbl. 283.05001.

9. H. S. M. Coxeter, The classification of zonohedra by means of projective diagrams, J. Math. Pures Appl. 41 (1962), 137-156. MR 25 \#417: Zbl. 123, 137. Reprinted with corrections in H. S. M. Coxeter, Twelve geometric essal's, Southern Illinois Univ. Press, Carbondale, Ill., 1968, pp. 54-74.

10. Henry H. Crapo, A higher invariant for matroids, J. Combin. Theory 2 (1967), 406-417. MR 35 \#6579: Zbl. 168, 262.

11. Jürgen Eckhoff, Radon's theorem revisited. Contributions to Geometry (Proc. Geom. Sympos.. Siegen, 1978), Birkhäuser, Basel, 1979. pp. 164-185. MR 81f: 52016: Zbl. 445.52009.

12. Jon Folkman and Jim Lawrence, Oriented matroids. J. Combin. Theory Ser. B 25 (1978), 199-236. MR 81g: 05045: Zbl. 325.05019 (382.05020).

13. C. Greene, Acyclic orientations (Notes), Higher Combinatorics (Proc. NATO Adv. Study Inst., Berlin, 1976), Reidel, Dordrecht, 1977. pp. 65-68. Zbl. 389.05036.

14. Jeanne W. Kerr and John E. Wetzel, Dissections of a triangular prism, Geometriae Dedicata 4 (1975), 279-289. MR 53 2714: Zbl. 335.05026.

15. Michel Las Vergnas, Matroïdes orientables, C. R. Acad. Sci. Paris Sér. A 280 (1975), A61-A64. MR 51 \# 7910; Zbl. 304.05013.

16. Acyclic and totally cyclic orientations of combinatorial geometries, Discrete Math. 20 (1977), 51-61. MR 57 2957; Zbl. 404.05017.

17. Sur les activités des orientations d'une géomiétrie combinatoire, Colloque Mathématiques Discrètes: Codes et Hypergraphes (Bruxelles, 1978), Cahiers Centre Études Rech. Opér. 20 (1978), 293-300. MR 81d: 05019; Zbl. 404.05018.

18. Convexity in oriented matroids, J. Combin. Theory Ser. B 29 (1980), 231-243. MR 82f: 05027: Zbl. 443.05026.

19. P. McMullen, On zonotopes, Trans. Amer. Math. Soc. 159 (1971), 91-109. Zbl. 223.52007.

$20 . \quad$ Transforms, diagrams and representations, Contributions to Geometry (Proc. Geom. Sympos., Siegen, 1978), Birkhäuser, Basel, 1979, pp. 92-130. MR 81i: 52007; Zbl. 445.52006.

21. Alfréd Rényi, Théorie des éléments saillants d'une suite d'observations, Colloquium on Combinatorial Methods in Probability Theory (Aarhus, 1962), Mat. Inst. Aarhus Univ., Aarhus, Denmark, n.d., pp. 104-117. Zbl. 139, 353. See also Actes du Colloq. de Math. Réuni à Clermont à l'Occasion du Tricentenaire de la Mort de Blaise Pascal, Tome II. Ann. Fac. Sci. Univ. Clermont-Ferrand No. 8 (1962), 7-13. MR 44 \#3376.

22. Gian-Carlo Rota, On the foundations of combinatorial theory. I. Theory of Möbius functions, $\mathrm{Z}$. Wahrsch. 2 (1964), 340-368. MR 30 4688; Zbl. 121, 24

23. Richard P. Stanley, Acyclic orientations of graphs, Discrete Math. 5(1973), 171-178. MR 47 \#6537; Zbl. 258.05113 .

24. Thomas Zaslavsky, Facing up to arrangements: Face-count formulas for partitions of space by hyperplanes, Mem. Amer. Math. Soc. 1 (1975), No. 154. MR 50 \# 9603; Zbl. 296.50010.

25. Counting the faces of cut-up spaces, Bull. Amer. Math. Soc. 81 (1975), 916-918. MR 53 \#3901; Zbl. 317.05012.

26. A A combinatorial analysis of topological dissections, Adv. in Math. 25 (1977), 267-285. MR 56 \#310; Zbl. 406.05004. 
27. __ Arrangements of hyperplanes; matroids and graphs, Proc. Tenth Southeastern Conf. on Combinatorics, Graph Theory and Computing (Boca Raton, 1979), Vol. II, Utilitas Math., Winnipeg, Man., 1979, pp. 895-911. MR 81h: 05043; Zbl. 434.05023.

28. Signed graphs, Discrete Appl. Math. 4 (1982), 47-74. Zbl. 476.05080. Erratum, ibid. 5 (1983), 248.

29. Orientation of signed graphs, (submitted).

30. Signed graph coloring, Discrete Math. 39 (1982), 215-228. Zbl. 487.05027.

Department of Mathematics, Haverford College, Haverford, Pennsylvania 19041

Department of Mathematics, Ohio State University, Columbus, Ohio 43210 